REALA, número 13, abril-septiembre de 2020

Sección: COMUNICACIONES Y COMENTARIOS JURISPRUDENCIALES

Recibido: 28-10-2019

Modificado: 26-02-2020

Aceptado: 04-03-2020

DOI: 10.24965/reala.i13.10746

Páginas: 131-151

\title{
El Impuesto sobre Transmisiones Patrimoniales y Actos Jurídicos Documentados y el ciclo político: incidencia económica desde una doble perspectiva
}

\section{The Tax on Capital Transfers and Documented Legal Acts and the political cycle: economic incidence from a double perspective}

\author{
M. ${ }^{a}$ Ángeles Ortega Almón \\ Universidad de Granada (España) \\ ORCID: https://orcid.org/0000-0003-0374-637X \\ maortega@ugr.es
}

\section{NOTA BIOGRÁFICA}

Profesora Titular del Departamento de Economía Aplicada de la Universidad de Granada. Sus líneas de investigación se centran en el campo de la economía del sector público, y más específicamente en: empresa pública y privatizaciones; financiación autonómica; impuestos medioambientales e Imposición sobre la riqueza.

Teresa Jesús Vílchez Ortiz Universidad de Granada (España) tevior@ugr.es

\section{NOTA BIOGRÁFICA}

Responsable de Negociado del CSIRC-Administración, Servicio Central de Informática de la Universidad de Granada. Máster en Dirección y Gestión Pública de la Universidad de Granada. Su línea de investigación se centra en la imposición sobre la riqueza.

Araceli Rojo Gallego-Burin

Universidad de Granada (España)

ORCID: https://orcid.org/0000-0001-8714-3693

gallegoburin@ugr.es

\section{NOTA BIOGRÁFICA}

Profesora de Economía y Organización de Empresas en la Universidad de Granada. Doctora en Ciencias Económicas y Empresariales por la Universidad de Granada. Acreditada como profesora ayudante doctora. Licenciada en Derecho, Administración y Dirección de Empresas e Investigación y Técnicas de Mercado. Sus líneas de investigación se centran en: Imposición medioambiental y sobre la riqueza; la gestión de proveedores.

\section{RESUMEN}

El Impuesto sobre Transmisiones Patrimoniales y Actos Jurídicos Documentados (ITPAJD) es un impuesto que grava la riqueza. Su recaudación está cedida a las CC.AA que tienen capacidad normativa para modificar los elementos básicos que determinan la deuda tributaria del contribuyente. Este trabajo pretende realizar una comparación de la normativa propia de las CC.AA de Régimen Común del Impuesto 
REALA. Nueva Época - N. ${ }^{13}$, abril-septiembre 2020 - ISSN: 1989-8975 - DOI: 10.24965/reala.i13.10746 - [Págs. 131-151]

El impuesto sobre transmisiones patrimoniales y actos jurídicos documentados y el ciclo político: incidencia económica desde ...

M. ${ }^{a}$ Ángeles Ortega Almón / Teresa Jesús Vílchez Ortiz / Araceli Rojo Gallego-Burin

(modalidades TPO y AJD), analizando la evolución de los tipos impositivos y bonificaciones con el objetivo de contrastar su evolución durante los periodos pre-electorales y post-electorales, en función de la configuración política del parlamento de cada comunidad autónoma. Asimismo, se analiza su incidencia económica desde una doble perspectiva, la del sujeto activo y la del sujeto pasivo del Impuesto. Es decir, tanto su incidencia presupuestaria en cada comunidad autónoma, como la incidencia sobre la deuda tributaria en función de la residencia de los contribuyentes, con el fin de demostrar las desigualdades territoriales que puedan generar dicho tributo (en sus dos modalidades) y la posible competencia fiscal entre comunidades, dependiendo del ciclo político.

\title{
PALABRAS CLAVE
}

ITPAJD; tipos impositivos; bonificaciones; deuda tributaria; CC.AA; ciclo político; recaudación.

\begin{abstract}
The Tax on Capital Transfers and Documented Legal Acts (TCTDLA) is a Spanish type of wealth tax. Its tax income is transferred to the Autonomous Communities, which have the regulatory capacity to modify the basic determinants of the taxpayer's tax debt. In this work, a comparison is made of the TCTDLA regulations (specifically its tax credits and tax rates) in each Autonomous Community with the aim of analysing their evolution during the pre-electoral and post-electoral periods, taking into account the political configuration of each Autonomous Parliament. Likewise, its economic impact is analyzed from the taxable person perspective and also from the active subject one. That is to say, its budgetary impact in each Autonomous Community is analyzed, as well as its incidence on taxpayer's tax debt, according to their tax domicile, in order to demonstrate the territorial inequalities that can generate said tax and the possible tax competition between Communities, depending on the political cycle.
\end{abstract}

\section{KEYWORDS}

Tax on Capital Transfers and Documented Legal Acts; tax rates; tax credits; tax debt; Autonomous Communities; political cycle; tax collection.

\section{SUMARIO}

INTRODUCCIÓN. 1. CARACTERÍSTICAS GENERALES DEL ITPAJD. 2. INCIDENCIA PRESUPUESTARIA DEL ITPAJD EN LAS CC.AA DE RÉGIMEN COMÚN. 3. EL ITPAJD Y LA DEUDA TRIBUTARIA DEL SUJETO PASIVO POR CC.AA DE RÉGIMEN COMÚN: ¿DESIGUALDADES TERRITORIALES? 4. EL ITPAJD Y EL CICLO POLÍTICO EN LAS CC.AA DE RÉGIMEN COMÚN. CONCLUSIONES. ANEXO I. RESULTADOS ELECTORALES DE LAS CC.AA DE RÉGIMEN COMÚN (2011-2018). REFERENCIAS BIBLIOGRÁFICAS.

\section{INTRODUCCIÓN}

Los impuestos que gravan la riqueza, afectan de forma sensible a dos importantes objetivos económicos: la redistribución de la renta y de la riqueza y la formación de capital. En España, dicha recaudación se realiza a través del Impuesto de Bienes Inmuebles (IBI, impuesto municipal), el Impuesto de Patrimonio (IP), el Impuesto sobre Sucesiones y Donaciones (ISD) y del Impuesto de Transmisiones Patrimoniales y Actos Jurídicos Documentados (ITPAJD). De estos tres últimos tributos, las CC.AA tienen cedidas capacidades normativas y aspectos básicos determinantes de la deuda tributaria (mínimos exentos, tipos impositivos, deducciones y bonificaciones), lo que ha generado una disparidad normativa que, a su vez, está dando dado lugar a notorias desigualdades entre los ciudadanos a la hora de tributar, según sea su lugar de residencia.

El Impuesto de Transmisiones Patrimoniales y Actos Jurídicos Documentados es un impuesto que grava las transmisiones de bienes y derechos inter vivos, complementando así al Impuesto sobre el Valor Añadido (IVA), con la diferencia de que el IVA se aplica cuando la persona que transmite el bien o derecho es una empresa o profesional, y el ITPAJD cuando se transmite entre particulares. Es un tributo de naturaleza indirecta que grava tres modalidades de actos jurídicos o contratos liquidables: las Transmisiones Patrimoniales Onerosas (TPO), los Actos Jurídicos Documentados (AJD) y las Operaciones Societarias (OS). De los dos primeros (TPO y AJD), las CC.AA tienen competencias normativas. 
Los efectos de la persistente crisis económica de estos últimos años, junto con la caída de la recaudación provocada por éstos, unido todo ello a la necesidad de cumplir con el objetivo de déficit, ha llevado a algunas Comunidades a incrementar los tipos impositivos en algunas modalidades, a la vez que otras los disminuían o los mantenían en el tipo estatal, dependiendo en ambos casos, tal como se quiere demostrar a lo largo de este trabajo, de intereses electoralistas provocados por los ciclos políticos.

El objetivo principal de esta investigación, es realizar una comparación de la normativa propia de las CC.AA de régimen común del Impuesto de Transmisiones Patrimoniales y Actos Jurídicos Documentados, en las dos modalidades (TPO y AJD) cedidas a las CC.AA, y ver cómo ha evolucionado ésta en los elementos básicos determinantes de la deuda tributaria del contribuyente, según la Comunidad, contrastando su evolución durante los periodos pre-electorales y post-electorales, de acuerdo con la configuración política del Parlamento de cada Comunidad. Asimismo, se pretende analizar y comparar el resultado en la carga tributaria de dichos impuestos condicionada por los tipos impositivos, bonificaciones y deducciones, propias de cada Comunidad Autónoma, en función del lugar de residencia de los sujetos pasivos, con el fin de demostrar las desigualdades territoriales que pueda generar su aplicación, y la posible competencia fiscal entre Comunidades, dependiendo del ciclo político.

En definitiva, se analiza la incidencia económica de este impuesto desde dos perspectivas, la del sujeto activo (CC.AA) y la del sujeto pasivo (contribuyente). Desde la perspectiva del sujeto activo, es decir, su incidencia presupuestaria, se analiza la evolución de la recaudación de este impuesto y su peso sobre los tributos no sujetos a liquidación y sobre la recaudación total de las CC.AA de régimen común, desde unos años antes del estallido de la burbuja inmobiliaria hasta el año 2016, último disponible. Para analizar la incidencia económica desde la perspectiva del contribuyente, se plantean dos supuestos prácticos (sobre el ITPO y el IAJD) que tratan de poner de manifiesto las diferentes cargas contributivas que un mismo sujeto pasivo debe de afrontar, por un mismo hecho imponible, según su lugar de residencia. Por último, se lleva a cabo un estudio comparativo, donde se pretende comprobar la relación de los cambios políticos y la incidencia tributaria que éstos han provocado en las distintas CC.AA. Para finalizar, se abordan las conclusiones principales del trabajo. Se ha incluido un Anexo que recoge la composición parlamentaria de las CC.AA de régimen común, así como los gobiernos resultantes de las convocatorias de elecciones autonómicas celebradas entre los años 2011 y 2018.

Las principales fuentes de información que se han utilizado para la realización de este trabajo son: las Memorias de la Administración Tributaria y de la Intervención General de la Administración del Estado, las Cuentas Generales de las CC.AA, la legislación estatal y autonómica del ITPAJD, las páginas webs de las Consejerías de Hacienda de las CC.AA de régimen común y la web de la Junta Electoral Central (JEC).

\section{CARACTERÍSTICAS GENERALES DEL ITPAJD}

El Impuesto sobre Transmisiones Patrimoniales y Actos Jurídicos Documentados se encuentra regulado por el Real Decreto Legislativo 1/1993 ${ }^{1}$, de 24 de septiembre y desarrollado por el Real Decreto 828/1995², de 29 de mayo. Podríamos decir que en él existen tres impuestos diferentes agrupados en un solo título. Así nos encontramos con el Impuesto sobre Transmisiones Patrimoniales Onerosas (TPO), el Impuesto sobre Operaciones Societarias (OS) y el Impuesto sobre Actos Jurídicos Documentados (AJD). Dada la existencia de características comunes a los tres y por razones históricas, el legislador optó por la denominación de un solo título integrador. De ahí que a cada uno de ellos se le atribuya la denominación de «modalidades» del impuesto, distinguiendo así las tres formas de gravamen.

Se trata de un impuesto de naturaleza indirecta el cual se devenga en el momento de realizar la transacción. El ITPAJD es un Impuesto estatal cuyo rendimiento está cedido al $100 \%$ a las CC.AA, quienes tienen competencias normativas ${ }^{3}$ a la vez que reguladoras sobre la gestión y liquidación del mismo. Las CC.AA

1 Real Decreto Legislativo 1/1993, de 24 de septiembre, por el que se aprueba el Texto Refundido de la Ley del Impuesto sobre Transmisiones Patrimoniales y Actos Jurídicos Documentados. Publicado en el BOE núm. 251, de 20 de octubre de 1993. URL: https:// www.boe.es/buscar/act.php?id=BOE-A-1993-25359.

2 Real Decreto 828/1995, de 29 de mayo, por el que se aprueba el Reglamento del Impuesto sobre Transmisiones Patrimoniales y Actos Jurídicos Documentados. Publicado en el BOE núm. 148, de 22 de junio de 1995, páginas 18.762 a 18.786 (25 págs.). URL: https://www.boe.es/buscar/doc.php?id=BOE-A-1995-15071.

3 LOFCA: Ley Orgánica 8/1980, de 22 de septiembre, de financiación de las Comunidades Autónomas. 
REALA. Nueva Época - N. ${ }^{13}$, abril-septiembre 2020 - ISSN: 1989-8975 - DOI: 10.24965/reala.i13.10746 - [Págs. 131-151]

El impuesto sobre transmisiones patrimoniales y actos jurídicos documentados y el ciclo político: incidencia económica desde ...

M. ${ }^{a}$ Ángeles Ortega Almón / Teresa Jesús Vílchez Ortiz / Araceli Rojo Gallego-Burin

pueden aprobar deducciones y bonificaciones de la cuota y modificación de los tipos de gravamen. Cuando las Comunidades ejercen su poder normativo sobre este tributo, generalmente lo utilizan para aplicar un aumento del tipo de gravamen. Además suelen establecer un tipo reducido especial aplicable a determinadas categorías de los hechos imponibles.

Para el ITPAJD existe una tarifa estatal que cualquier Comunidad Autónoma puede utilizar para su liquidación, aunque en el marco de sus competencias, la mayoría de ellas han establecido unas tarifas propias aplicables a la liquidación del mismo.

En la tabla 1, se refleja, como ejemplo de ello, los distintos tipos de la modalidad de Transmisiones Patrimoniales que las Comunidades están aplicando en el 2019, según el objeto del bien o derecho a transmitir. Como se puede observar, existen notables diferencias entre las distintas Comunidades con respecto a los tipos impositivos que cada una de ellas aplica para los casos que se muestran en la tabla. Los tipos impositivos que más varían entre las Comunidades son los que se aplican a la transmisión de bienes inmuebles (así como en la constitución y cesión de derechos reales sobre los mismos, excepto los de garantía), que oscilan entre el $6 \%$ y el $11 \%$. Por ejemplo, mientras que la Comunidad de Madrid mantiene el tipo de gravamen que coincide con el de regulación estatal (6\%), las Comunidades de Andalucía, Aragón, Principado de Asturias, Islas Baleares, Cataluña y Extremadura aplican un tipo de gravamen progresivo en función del valor del inmueble, utilizando diferentes tramos. Canarias, Cantabria, Castilla y León, Castilla-La Mancha, Galicia, Región de Murcia, La Rioja y C. Valenciana han fijado tipos proporcionales superiores al $6 \%$ que establece la normativa estatal.

TABla 1. Tipos de gravamen en función de la modalidad del ITP POR CC.AA de RÉgimen Común, EN EL AÑo 2019

\begin{tabular}{|c|c|c|c|c|}
\hline \multirow[b]{2}{*}{$\begin{array}{l}\text { Comunidad } \\
\text { autónoma }\end{array}$} & \multicolumn{4}{|c|}{ Transmisiones Patrimoniales Onerosas } \\
\hline & $\begin{array}{l}\text { Transmisión } \\
\text { de Bienes } \\
\text { Inmuebles }\end{array}$ & $\begin{array}{c}\text { Transmisión } \\
\text { de Bienes Muebles } \\
\text { y Semovientes }\end{array}$ & $\begin{array}{l}\text { Concesiones } \\
\text { Administrativas }\end{array}$ & $\begin{array}{l}\text { Arrendamientos } \\
\text { de Muebles } \\
\text { e Inmuebles }\end{array}$ \\
\hline ESTATAL & $6 \%$ & $4 \%$ & $4 \%$ & Tabla progresiva \\
\hline Andalucía & Del $8 \%$ al $10 \%$ & $E$ & $E$ & $E$ \\
\hline Aragón & Del $8 \%$ al $10 \%$ & $E$ & $E$ & $E$ \\
\hline P. de Asturias & Del $8 \%$ al $10 \%$ & $E$ & $E$ & $E$ \\
\hline I. Baleares & Del $8 \%$ al $11 \%$ & $1 \%$ & $\mathrm{E}$ & $E$ \\
\hline Canarias & $6,50 \%$ & $5,50 \%$ & $7,00 \%$ & $E$ \\
\hline Cantabria & $10 \%$ & $8 \%$ & $10 \%$ & Bonificación del 99\% \\
\hline Castilla y León & $8 \%$ & $5 \%$ & $7 \%$ & $E$ \\
\hline Castilla-La Mancha & $9 \%$ & $6 \%$ & $9 \%$ & $E$ \\
\hline Cataluña & Del $10 \%$ al $11 \%$ & $5 \%$ & $E$ & $E$ \\
\hline Extremadura & Del $8 \%$ al $11 \%$ & $6 \%$ & $8 \%$ & $E$ \\
\hline Galicia & $10 \%$ & $8 \%$ & $E$ & $E$ \\
\hline C. de Madrid & $6 \%$ & $E$ & $E$ & $E$ \\
\hline R. de Murcia & $8 \%$ & $E$ & $E$ & $E$ \\
\hline La Rioja & $7 \%$ & $4 \%$ & $7 \%$ & $E$ \\
\hline C. Valenciana & $10 \%$ & $6 \%$ & $E$ & $E$ \\
\hline
\end{tabular}

Fuente: Consejo General de Economistas (2019) y elaboración propia. Nota: $\mathrm{E}=$ Tarifa Estatal. 
REALA. Nueva Época - N. ${ }^{13}$, abril-septiembre 2020 - ISSN: 1989-8975 - DOI: 10.24965/reala.i13.10746 - [Págs. 131-151]

El impuesto sobre transmisiones patrimoniales y actos jurídicos documentados y el ciclo político: incidencia económica desde ... M. ${ }^{a}$ Ángeles Ortega Almón / Teresa Jesús Vílchez Ortiz / Araceli Rojo Gallego-Burin

\section{INCIDENCIA PRESUPUESTARIA DEL ITPAJD EN LAS CC.AA DE RÉGIMEN COMÚN}

Los recursos con los que cuentan las CC.AA para su financiación, con el vigente modelo de financiación autonómica, provienen de dos grupos de ingresos: fuentes tributarias y fuentes no tributarias. Dentro del primer grupo, se encuentran los tributos totalmente cedidos a las CC.AA para su gestión y recaudación como son el Impuesto de Sucesiones y Donaciones (ISD), el Impuesto sobre Transmisiones Patrimoniales y Actos Jurídicos Documentados (ITPAJD), el Impuesto sobre el Patrimonio (IP) cedido a las CC.AA a partir del 2012, los Impuestos Especiales sobre determinados Medios de Transporte y los tributos sobre el Juego.

En la Tabla 2 se recoge la actividad recaudatoria del ITPAJD en las CC.AA de régimen común, en el año 2017, último disponible, así como el peso que dicho tributo representa sobre el total de los tributos cedidos, y sobre los Ingresos Tributarios Totales de éstas, en ese mismo año.

TABLA 2. Recaudación POR CC.AA DEL ITPAJD EN EL AÑo 2017 Y PESO DE DiCHO IMPUESTO SOBRE EL TOTAL DE LOS TRIBUTOS CEDIDOS Y SOBRE EL TOTAL DE LA RECAUDACIÓN TRIBUTARIA (EN MILES DE EUROS Y EN PORCENTAJES)

\begin{tabular}{|c|c|c|c|c|c|c|}
\hline \multirow{2}{*}{$\begin{array}{l}\text { Comunidad } \\
\text { autónoma }\end{array}$} & \multicolumn{2}{|c|}{ ITPAJD } & \multirow{2}{*}{$\begin{array}{c}\text { Total } \\
\text { tributos } \\
\text { cedidos }\end{array}$} & \multirow{2}{*}{$\begin{array}{c}\text { Total } \\
\text { tributos }\end{array}$} & \multirow{2}{*}{$\begin{array}{l}\text { Peso del } \\
\text { ITPAJD sobre } \\
\text { los tributos } \\
\text { Cedidos }\end{array}$} & \multirow{2}{*}{$\begin{array}{l}\text { Peso del } \\
\text { ITPAJD sobre } \\
\text { los tributos } \\
\text { totales }\end{array}$} \\
\hline & TPO & AJD & & & & \\
\hline Andalucía & 1.058 .358 & 389.809 & 2.045 .084 & $14.315 .509,01$ & 70,81 & 10,12 \\
\hline Aragón & 113.382 & 56.243 & 425.795 & $3.289 .798,05$ & 39,84 & 5,16 \\
\hline P. de Asturias & 83.909 & 26.401 & 253.101 & $2.286 .427,72$ & 43,58 & 4,82 \\
\hline I. Baleares & 508.465 & 110.205 & 816.631 & $3.204 .518,99$ & 75,76 & 19,31 \\
\hline Canarias & 235.564 & 61.770 & 425.648 & $1.751 .472,31$ & 69,85 & 16,98 \\
\hline Cantabria & 71.002 & 22.519 & 162.351 & $1.365 .645,69$ & 57,60 & 6,85 \\
\hline Castilla y León & 182.464 & 75.155 & 544.103 & $5.270 .973,92$ & 47,35 & 4,89 \\
\hline Castilla-La Mancha & 198.617 & 89.642 & 417.473 & $3.698 .799,66$ & 69,05 & 7,79 \\
\hline Cataluña & 1.474 .235 & 459.187 & 3.069 .452 & $20.265 .576,56$ & 62,99 & 9,54 \\
\hline Extremadura & 64.056 & 28.568 & 158.087 & $1.736 .395,47$ & 58,59 & 5,33 \\
\hline Galicia & 178.690 & 73.030 & 511.267 & $5.515 .424,03$ & 49,23 & 4,56 \\
\hline C. de Madrid & 1.005 .160 & 366.616 & 1.928 .933 & $19.139 .563,35$ & 71,12 & 7,17 \\
\hline R. de Murcia & 132.420 & 60.940 & 306.702 & $2.641 .672,78$ & 63,04 & 7,32 \\
\hline La Rioja & 26.231 & 6.204 & 68.551 & $690.355,23$ & 47,32 & 4,70 \\
\hline C. Valenciana & 826.815 & 225.009 & 1.585 .980 & $10.410 .142,41$ & 66,32 & 10,10 \\
\hline Total & 6.159 .368 & 2.051.298 & 12.719 .158 & $95.582 .275,19$ & 64,55 & 8,59 \\
\hline
\end{tabular}

Fuente: Ministerio de Hacienda y Función Pública y elaboración propia.

La recaudación del ITPAJD en el conjunto de CC.AA tiene un gran peso sobre la recaudación de los tributos cedidos, constituyendo un $64,55 \%$ del total de dicha recaudación, en el año 2017 . Este alto porcentaje tiene su principal protagonista en la modalidad de TPO, la cual supone casi la mitad de la recaudación de los Impuestos Cedidos. Sin embargo, su importancia sobre la recaudación tributaria total es bastante más modesta, representando solo el $8,59 \%$ de la recaudación total.

La Comunidad que más recauda por este impuesto es Cataluña (1.933,42 millones de euros) seguida por Andalucía (1.448,17 millones de euros). Entre las que menos ingresa está La Rioja, con una recaudación de 32,435 millones de euros, seguida por Extremadura con 92,62 millones de euros. Las Comunidades que mayor peso han tenido sobre la recaudación de los tributos cedidos han sido Islas Baleares y Comunidad 
de Madrid $(75,76 \%$ y $71,12 \%$, respectivamente), frente a Aragón que ha sido la que representa el menor peso con un 39,84\%. Respecto al peso sobre la recaudación total, las Islas Baleares (19,31\%) encabezan la recaudación, y en el lado opuesto, se encuentra Galicia, con solo un peso del $4,56 \%$.

En la Figura 1, se puede apreciar la evolución comparativa del ITPAJD, desglosando y diferenciando la recaudación de este tributo, en la modalidad de Transmisiones Patrimoniales y la modalidad de Actos Jurídicos Documentados, así como del total de los tributos no sujetos a liquidación y del total de los tributos cedidos (total o parcialmente) a las CC.AA de régimen común, durante el periodo 2006 a 2016, en euros constantes y utilizando como año base 2015.

Tanto la recaudación de los tributos cedidos no sujetos a liquidación como la de los impuestos en la modalidad de TP y AJD siguen una evolución paralela de descenso, tocando fondo en el 2009 la modalidad de AJD y TP en 2011, como se puede observar, de forma clara, en la Figura 1. Como se aprecia, los periodos de recuperación en la recaudación tributaria, tienen como claros protagonistas a los tributos sujetos a liquidación, los cuales han servido de multiplicadores de dicha recaudación pero de forma heterogénea, en unos años de plena crisis económica y en los que el efecto que se esperaría de ellos es totalmente contrario a lo acaecido. Sin embargo, a partir del año 2009 aumentan el total de recursos tributarios del conjunto de CC.AA de régimen común. Este protagonismo de los tributos sujetos a liquidación, en este período inmediato posterior a la crisis, tiene su origen en la reforma del sistema de financiación de las CC.AA de régimen común que tuvo lugar en el 2009, y cuyo marco legislativo se materializa en la Ley 22/2009 de 18 de diciembre ${ }^{4}$, siendo el que está vigente hoy día.

FIgURA 1. EVOLUCIÓN COMPARATIVA DE LA RECAUDACIÓN DE ITP, IAJD, TRIBUTOS NO SUJETOS A LIQUIDACIÓN Y POR TRIBUTOS TOTALES CEDIDOS A LAS CC.AA (2006-2016) (EUROS CONSTANTES 2015)

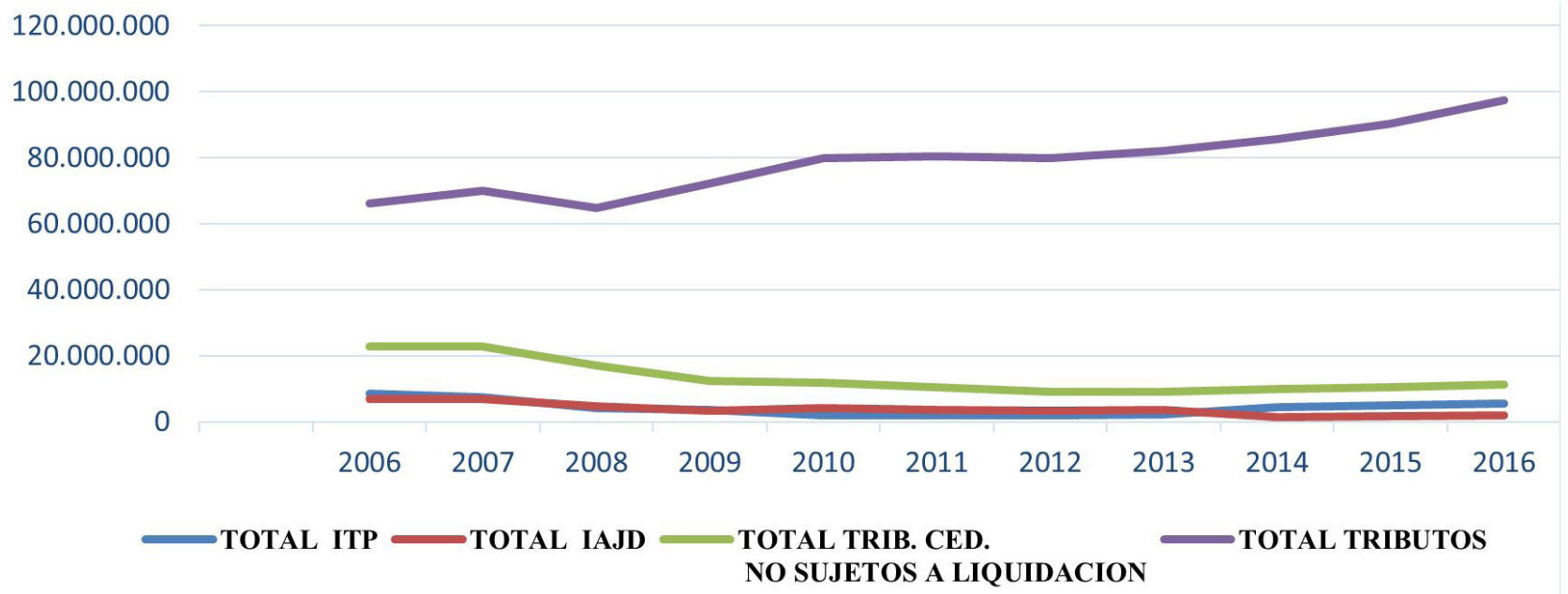

Fuente: Ministerio de Hacienda y elaboración propia.

El actual modelo comenzó a surtir efectos el 1 de enero de 2009, en plena recesión, pero con una dotación generosa, que se financió vía endeudamiento. El nuevo modelo pretendía mayor corresponsabilidad fiscal, para lo cual se aumentaron los porcentajes de cesión de los tributos parcialmente cedidos a las Comunidades Autónomas, al tiempo que se incrementaron sus competencias normativas para reforzar su autonomía financiera. De este modo, si con el modelo anterior el $70 \%$ de todos los recursos de las regiones procedía de los impuestos cedidos parcial o totalmente por el Estado, con el nuevo sistema, este porcentaje pasó a rondar el $90 \%$.

Con el nuevo modelo de financiación de 2009, el porcentaje de cesión a las CC.AA del IRPF pasa del $33 \%$ al $50 \%$; en el IVA pasa del $35 \%$ al $50 \%$. De igual forma, los Impuestos Especiales de Fabricación (Cerveza, Vino y bebidas fermentadas, Productos Intermedios, Alcohol y Bebidas Derivadas, Labores del Tabaco e Hidrocarburos) pasan del $40 \%$ de cesión al $58 \%$. El aumento del porcentaje de cesión del IVA tuvo

4 Ley 22/2009, de 18 de diciembre, por la que se regula el sistema de financiación de las Comunidades Autónomas de régimen común y Ciudades con Estatuto de Autonomía y se modifican determinadas normas tributarias. URL: https://www.boe.es/buscar/act. php?id=BOE-A-2009-20375. 
REALA. Nueva Época - N. ${ }^{13}$, abril-septiembre 2020 - ISSN: 1989-8975 - DOI: 10.24965/reala.i13.10746 - [Págs. 131-151]

El impuesto sobre transmisiones patrimoniales y actos jurídicos documentados y el ciclo político: incidencia económica desde ...

M. ${ }^{a}$ Ángeles Ortega Almón / Teresa Jesús Vílchez Ortiz / Araceli Rojo Gallego-Burin

un segundo efecto sobre la recaudación de las CC.AA el 1 de septiembre de 2012, cuando se incrementó el tipo general del IVA pasando del $18 \%$ al $21 \%$, el tipo reducido pasó del $8 \%$ al $10 \%$, dejando el tipo superreducido en el $4 \%$, aunque algunos grupos de productos, pasaron del tipo reducido o superreducido al general, como por ejemplo, el material escolar, que se elevó al $21 \% 5$.

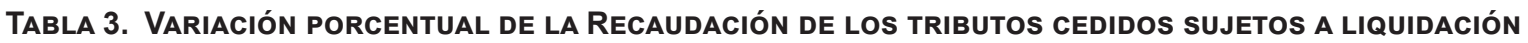
Y DEL ITPAJD (2008-2016) (PORCENTAJES)

\begin{tabular}{lcccccccc}
\hline \multicolumn{1}{c}{ Períodos } & $\mathbf{2 0 0 8 - 2 0 0 9}$ & $\mathbf{2 0 0 9 - 2 0 1 0}$ & $\mathbf{2 0 1 0 - 2 0 1 1}$ & $\mathbf{2 0 1 1 - 2 0 1 2}$ & $\mathbf{2 0 1 2 - 2 0 1 3}$ & $\mathbf{2 0 1 3 - 2 0 1 4}$ & $\mathbf{2 0 1 4 - 2 0 1 5}$ & $\mathbf{2 0 1 5 - 2 0 1 6}$ \\
\hline VAR IVA & $-0,14$ & 46,24 & 0,44 & 2,36 & 2,91 & 8,17 & 7,35 & 4,21 \\
\hline VAR IRPF & 40,73 & $-2,71$ & 0,68 & $-5,63$ & 1,07 & 2,61 & 5,77 & 4,56 \\
\hline VAR IIEE & 37,66 & 2,57 & $-3,93$ & $-3,38$ & 5,72 & 1,22 & 1,81 & 1,19 \\
\hline VAR ITPAJD & 32,31 & 10,88 & $-0,22$ & $-2,70$ & 1,51 & 4,55 & 6,37 & 2,18 \\
\hline
\end{tabular}

Fuente: Ministerio de Hacienda y elaboración propia.

Por tanto, se puede considerar que en el periodo posterior a la crisis, la importancia recaudatoria de los tributos cedidos no sujetos a liquidación y más específicamente el ITPAJD ha sido mínima, en comparación con la obtenida por los impuestos sujetos a liquidación. Así en 2006, el ITPAJD constituía el $22,79 \%$ del total de la recaudación por tributos, mientras que en 2016 se comprueba como el ITPAJD constituye solamente un $7,60 \%$ de la recaudación tributaria total conseguida por las CC.AA de régimen común.

\section{EL ITPAJD Y LA DEUDA TRIBUTARIA DEL SUJETO PASIVO POR CC.AA DE RÉGIMEN COMÚN: ¿DESIGUALDADES TERRITORIALES?}

Con el objetivo de mostrar las diferencias más significativas que se producen en el pago del Impuesto sobre Transmisiones Patrimoniales y Actos Jurídicos Documentados, al que se sometería un mismo sujeto pasivo según la Comunidad Autónoma con competencia para recaudar éste y, su evolución en la misma Comunidad en el periodo analizado, a continuación, se plantean varios supuestos prácticos, seleccionando de las dos modalidades posibles del Impuesto, las que tienen una mayor incidencia recaudatoria de este tributo: Transmisiones Patrimoniales inter vivos y en el caso de la modalidad de Actos Jurídicos Documentados, la constitución de una hipoteca.

En cuanto al pago del Impuesto de Transmisiones Patrimoniales inter vivos se parte del supuesto en el que «una ciudadana de 42 años adquiere su primera vivienda habitual de segunda mano a un particular, con un valor real de 150.000 euros».

En la Tabla 4 se recogen los tipos impositivos aplicados a las Transmisiones Patrimoniales de inmuebles, materializándose en la Tabla 5, la deuda tributaria que ha de afrontar un mismo sujeto pasivo, según la Comunidad Autónoma dónde se sitúe su domicilio fiscal y cómo evoluciona dicha deuda a lo largo del periodo analizado.

En las tablas 4 y 5 se puede observar que en el año 2009, casi todas las Comunidades Autónomas de régimen común tenían establecido el mismo tipo impositivo (7\%) en la modalidad de Transmisiones Patrimoniales de inmuebles, correspondiéndole una deuda tributaria de $10.500 €$. En consecuencia en dicho año no se aprecian desigualdades territoriales impositivas en la modalidad del TPO entre las distintas Comunidades. Sin embargo, es en el año 2013 donde se pueden observar más cambios, ya que se da un aumento en los tipos impositivos y por tanto, en las cuantías de la deuda tributaria a pagar por los sujetos pasivos, en dos tercios de las CC.AA. Este incremento de los tipos impositivos puede deberse a que en el 2013, la complicada situación presupuestaria y consecuente merma de ingresos en las arcas públicas, como consecuencia

\footnotetext{
5 Entre las subidas más llamativas encontramos: Viviendas, garajes y anexos en las que el tipo impositivo del IVA pasa del $4 \%$ al $10 \%$; Servicios funerarios; Entradas a espectáculos, zoológicos, conciertos, salas de cine, circos, parques de atracciones o Asistencia sanitaria, dental y curas termales, el tipo de gravamen sube del $8 \%$ al $21 \%$; las entradas a bibliotecas, archivos, museos, galerías de arte pasa del $8 \%$ al $10 \%$.
} 
REALA. Nueva Época - N. ${ }^{13}$, abril-septiembre 2020 - ISSN: 1989-8975 - DOI: 10.24965/reala.i13.10746 - [Págs. 131-151]

El impuesto sobre transmisiones patrimoniales y actos jurídicos documentados y el ciclo político: incidencia económica desde ... M. ${ }^{a}$ Ángeles Ortega Almón / Teresa Jesús Vílchez Ortiz / Araceli Rojo Gallego-Burin

de la gran recesión económica, padecida desde el año 2008, con ello se pretendía corregir o amortiguar el descenso en la recaudación y su impacto en el déficit público de dichas Comunidades.

Tabla 4. Evolución del tipo impositivo aplicado a las TRANSmisiones PatRimoniales de InMUebles POR CC.AA (2009-2019) (PORCENTAJES)

\begin{tabular}{|c|c|c|c|c|c|c|c|c|c|c|c|}
\hline CC.AA/Años & 2009 & 2010 & 2011 & 2012 & 2013 & 2014 & 2015 & 2016 & 2017 & 2018 & 2019 \\
\hline Estatal & $6 \%$ & $6 \%$ & $6 \%$ & $6 \%$ & $6 \%$ & $6 \%$ & $6 \%$ & $6 \%$ & $6 \%$ & $6 \%$ & $6 \%$ \\
\hline Andalucía & $7 \%$ & $7 \%$ & $7 \%$ & $8 \%$ & $8 \%$ & $8 \%$ & $8 \%$ & $8 \%$ & $8 \%$ & $8 \%$ & $8 \%$ \\
\hline Aragón & $7 \%$ & $7 \%$ & $7 \%$ & $7 \%$ & $7 \%$ & $7 \%$ & $7 \%$ & $8 \%$ & $8 \%$ & $8 \%$ & $8 \%$ \\
\hline P. de Asturias & $7 \%$ & $7 \%$ & $8 \%$ & $8 / \%$ & $8 \%$ & $8 \%$ & $8 \%$ & $8 \%$ & $8 \%$ & $8 \%$ & $8 \%$ \\
\hline I. Baleares & $7 \%$ & $7 \%$ & $7 \%$ & $7 \%$ & $8 \%$ & $8 \%$ & $8 \%$ & $8 \%$ & $8 \%$ & $8 \%$ & $8 \%$ \\
\hline Canarias & $6,50 \%$ & $6,50 \%$ & $6,50 \%$ & $6,50 \%$ & $6,50 \%$ & $6,50 \%$ & $6,50 \%$ & $6,50 \%$ & $6,50 \%$ & $6,50 \%$ & $6,50 \%$ \\
\hline Cantabria & $7 \%$ & $7 \%$ & $7 \%$ & $7 \%$ & $8 \%$ & $8 \%$ & $8 \%$ & $8 \%$ & $8 \%$ & $10 \%$ & $9 \%^{a}$ \\
\hline Castilla y León & $7 \%$ & $7 \%$ & $7 \%$ & $7 \%$ & $8 \%$ & $8 \%$ & $8 \%$ & $8 \%$ & $8 \%$ & $8 \%$ & $8 \%$ \\
\hline Castilla-La Mancha & $7 \%$ & $7 \%$ & $7 \%$ & $7 \%$ & $8 \%$ & $8 \%$ & $8 \%$ & $8 \%$ & $9 \%$ & $9 \%$ & $9 \%$ \\
\hline Cataluña & $7 \%$ & $7 \%$ & $8 \%$ & $8 \%$ & $8 \%$ & $10 \%$ & $10 \%$ & $10 \%$ & $10 \%$ & $10 \%$ & $10 \%$ \\
\hline Extremadura & $7 \%$ & $7 \%$ & $7 \%$ & $7 \%$ & $8 \%$ & $8 \%$ & $8 \%$ & $8 \%$ & $8 \%$ & $8 \%$ & $8 \%$ \\
\hline Galicia & $7 \%$ & $7 \%$ & $7 \%$ & $7 \%$ & $10 \%$ & $10 \%$ & $10 \%$ & $10 \%$ & $10 \%$ & $10 \%$ & $10 \%$ \\
\hline C. de Madrid & $7 \%$ & $7 \%$ & $7 \%$ & $7 \%$ & $7 \%$ & $6 \%$ & $6 \%$ & $6 \%$ & $6 \%$ & $6 \%$ & $6 \%$ \\
\hline R. de Murcia & $7 \%$ & $7 \%$ & $7 \%$ & $7 \%$ & $8 \%$ & $8 \%$ & $8 \%$ & $8 \%$ & $8 \%$ & $8 \%$ & $8 \%$ \\
\hline La Rioja & $7 \%$ & $7 \%$ & $7 \%$ & $7 \%$ & $7 \%$ & $7 \%$ & $7 \%$ & $7 \%$ & $7 \%$ & $7 \%$ & $7 \%$ \\
\hline C. Valenciana & $7 \%$ & $7 \%$ & $7 \%$ & $7 \%$ & $8 \%$ & $10 \%$ & $10 \%$ & $10 \%$ & $10 \%$ & $10 \%$ & $10 \%$ \\
\hline
\end{tabular}

a En las transmisiones de viviendas que vayan a constituir la vivienda habitual, la C. de Cantabria en 2019 introduce un tipo progresivo entre el $8 \%$ y el $10 \%$ según la Base Imponible.

Fuente: Elaboración propia.

En los siguientes años, los tipos impositivos van sufriendo algunas modificaciones, llegando así al año 2019 en el que ninguna Comunidad Autónoma mantiene el $7 \%$ del año 2009, salvo La Rioja, y con la peculiaridad de que la única Comunidad que incluso disminuye su tipo impositivo es la Comunidad de Madrid, estableciendo un $6 \%$.

En el año 2019 los tipos impositivos abarcan desde el $6 \%$ al $10 \%$, observándose como el tipo impositivo del $8 \%$ es el que se ha impuesto entre las Comunidades (concretamente siete de ellas). Los distintos tipos de gravamen llevan aparejadas variaciones en las cuotas tributarias a pagar, abarcando éstas desde $9.000 €$ hasta $15.000 €$. La diferencia existente entre ambas cantidades $(6.000 €)$ refleja la existencia de las desigualdades a las que ha de hacer frente un mismo sujeto pasivo por un mismo hecho imponible en función de la Comunidad en la que tenga su domicilio fiscal.

Tabla 5. Deuda Tributaria a pagar por un SUjeto pasivo en la modalidad de Transmisiones Patrimoniales DE INMUEBLES POR CC.AA (EN EUROS)

\begin{tabular}{lrrrrrrrrrrr}
\hline CC.AA/Años & $\mathbf{2 0 0 9}$ & $\mathbf{2 0 1 0}$ & $\mathbf{2 0 1 1}$ & $\mathbf{2 0 1 2}$ & $\mathbf{2 0 1 3}$ & $\mathbf{2 0 1 4}$ & $\mathbf{2 0 1 5}$ & $\mathbf{2 0 1 6}$ & $\mathbf{2 0 1 7}$ & $\mathbf{2 0 1 8}$ & $\mathbf{2 0 1 9}$ \\
\hline Estatal & 9.000 & 9.000 & 9.000 & 9.000 & 9.000 & 9.000 & 9.000 & 9.000 & 9.000 & 9.000 & 9.000 \\
\hline Andalucía & 10.500 & 10.500 & 10.500 & 12.000 & 12.000 & 12.000 & 12.000 & 12.000 & 12.000 & 12.000 & 12.000 \\
\hline
\end{tabular}


REALA. Nueva Época - N. ${ }^{\circ}$ 13, abril-septiembre 2020 - ISSN: 1989-8975 - DOI: 10.24965/reala.i13.10746 - [Págs. 131-151]

El impuesto sobre transmisiones patrimoniales y actos jurídicos documentados y el ciclo político: incidencia económica desde ... M. ${ }^{a}$ Ángeles Ortega Almón / Teresa Jesús Vílchez Ortiz / Araceli Rojo Gallego-Burin

\begin{tabular}{|c|c|c|c|c|c|c|c|c|c|c|c|}
\hline CC.AA/Años & 2009 & 2010 & 2011 & 2012 & 2013 & 2014 & 2015 & 2016 & 2017 & 2018 & 2019 \\
\hline Aragón & 10.500 & 10.500 & 10.500 & 10.500 & 10.500 & 10.500 & 10.500 & 12.000 & 12.000 & 12.000 & 12.000 \\
\hline P. de Asturias & 10.500 & 10.500 & 12.000 & 12.000 & 12.000 & 12.000 & 12.000 & 12.000 & 12.000 & 12.000 & 12.000 \\
\hline I. Baleares & 10.500 & 10.500 & 10.500 & 10.500 & 12.000 & 12.000 & 12.000 & 12.000 & 12.000 & 12.000 & 12.000 \\
\hline Canarias & 9.750 & 9.750 & 9.750 & 9.750 & 9.750 & 9.750 & 9.750 & 9.750 & 9.750 & 9.750 & 9.750 \\
\hline Cantabria & 10.500 & 10.500 & 10.500 & 10.500 & 12.000 & 12.000 & 12.000 & 12.000 & 12.000 & 15.000 & 13.500 \\
\hline Castilla y León & 10.500 & 10.500 & 10.500 & 10.500 & 12.000 & 12.000 & 12.000 & 12.000 & 12.000 & 12.000 & 12.000 \\
\hline Castilla-La Mancha & 10.500 & 10.500 & 10.500 & 10.500 & 12.000 & 12.000 & 12.000 & 12.000 & 13.500 & 13.500 & 13.500 \\
\hline Cataluña & 10.500 & 10.500 & 12.000 & 12.000 & 12.000 & 15.000 & 15.000 & 15.000 & 15.000 & 15.000 & 15.000 \\
\hline Extremadura & 10.500 & 10.500 & 10.500 & 10.500 & 12.000 & 12.000 & 12.000 & 12.000 & 12.000 & 12.000 & 12.000 \\
\hline Galicia & 10.500 & 10.500 & 10.500 & 10.500 & 15.000 & 15.000 & 15.000 & 15.000 & 15.000 & 15.000 & 15.000 \\
\hline C. de Madrid & 10.500 & 10.500 & 10.500 & 10.500 & 10.500 & 9.000 & 9.000 & 9.000 & 9.000 & 9.000 & 9.000 \\
\hline R. de Murcia & 10.500 & 10.500 & 10.500 & 10.500 & 12.000 & 12.000 & 12.000 & 12.000 & 12.000 & 12.000 & 12.000 \\
\hline La Rioja & 10.500 & 10.500 & 10.500 & 10.500 & 10.500 & 10.500 & 10.500 & 10.500 & 10.500 & 10.500 & 10.500 \\
\hline C. Valenciana & 10.500 & 10.500 & 10.500 & 10.500 & 12.000 & 15.000 & 15.000 & 15.000 & 15.000 & 15.000 & 15.000 \\
\hline
\end{tabular}

Fuente: Elaboración Propia.

Hay que resaltar el hecho de que una variación porcentual de un punto, signifique un aumento a pagar en la cuota tributaria de $1.500 €$, lo que supone un esfuerzo considerable para un sujeto pasivo con una renta media/baja. Respecto a esta circunstancia, hay que destacar el hecho de que en el ejercicio 2019 solamente una Comunidad Autónoma, en este caso Cantabria, rebaja su tipo impositivo en un punto porcentual.

Figura 2. Distribución de CUOTAS A PAGAR POR UN MISMO SUJETO PASIVO SEGÚN LA CC.AA EN LA MODALIDAD DE TRANSmisiones Patrimoniales de InMUebles EN EL AÑo (2019) (EN EUROS)

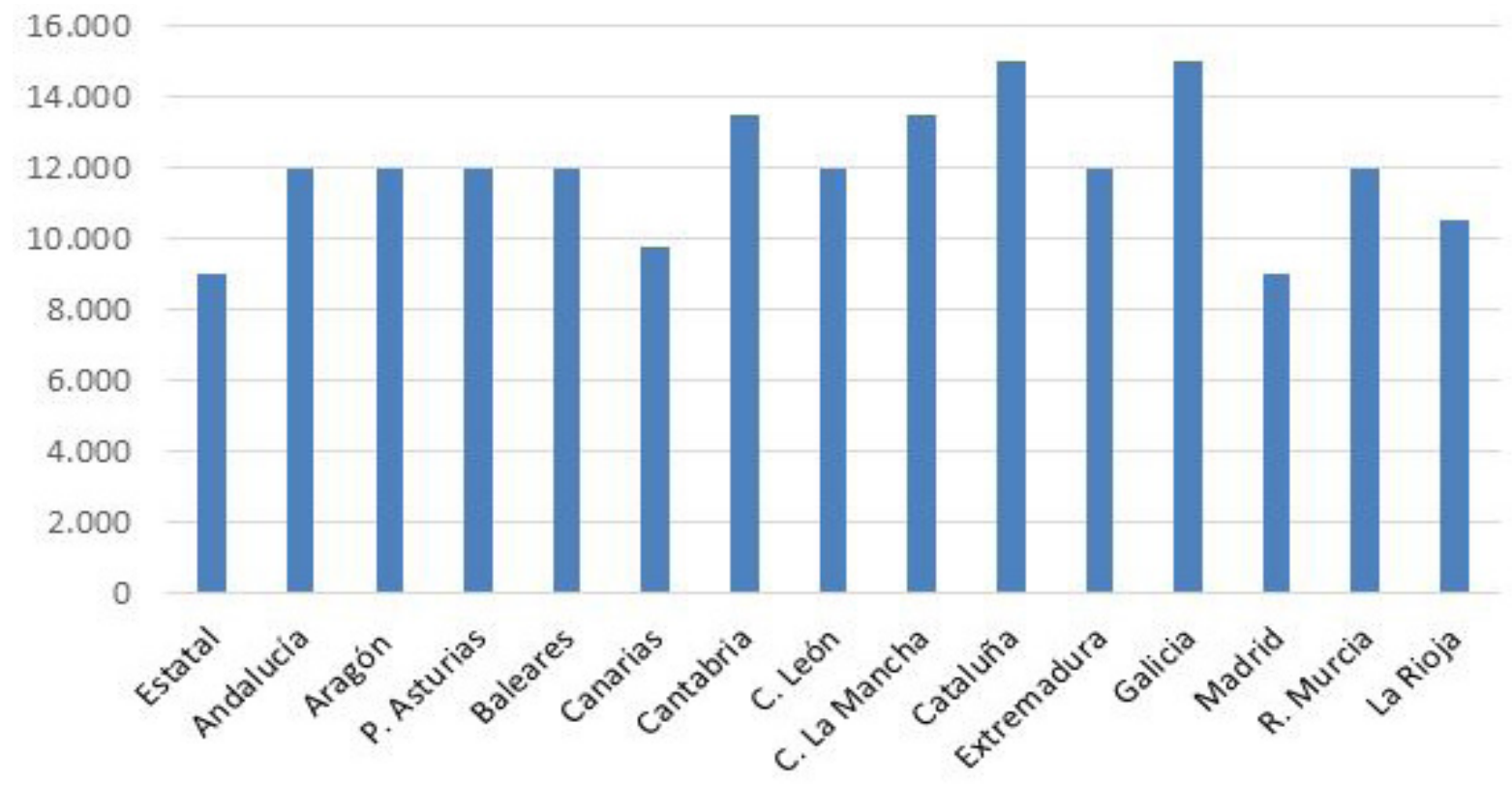

Fuente: Elaboración Propia. 
REALA. Nueva Época - N. ${ }^{13}$, abril-septiembre 2020 - ISSN: 1989-8975 - DOI: 10.24965/reala.i13.10746 - [Págs. 131-151]

El impuesto sobre transmisiones patrimoniales y actos jurídicos documentados y el ciclo político: incidencia económica desde ...

M. ${ }^{a}$ Ángeles Ortega Almón / Teresa Jesús Vílchez Ortiz / Araceli Rojo Gallego-Burin

Para el caso de la modalidad de Actos Jurídicos Documentados, se parte del supuesto en el que «una ciudadana de 42 años adquiere su primera vivienda habitual de segunda mano, a un particular, con un valor real de 150.000 euros con la consiguiente constitución de un préstamo hipotecario para dicha adquisición».

En la Tabla 6 se recogen los tipos impositivos aplicados a las Actos Jurídicos Documentados por la constitución de una hipoteca sobre la adquisición de un inmueble, materializándose en la Tabla 7 la deuda tributaria que ha de afrontar el sujeto pasivo por CC.AA y en el mismo periodo.

Como se puede observar, en la Tabla 6, en el año 2013 se da un aumento casi generalizado de los tipos de gravamen del IAJD, afectando a nueve de las quince CC.AA, al igual que ocurría con el ITP. En este año todas las CC.AA superaban el tipo Estatal en el doble o más, salvo Canarias. En el año 2014 se mantienen dichos tipos impositivos, salvo la Comunidad de Madrid que lo disminuye y la Comunidad Valenciana que lo aumenta en tres décimas porcentuales. En el año 2016, solamente Aragón aumenta medio punto porcentual, y en el 2017 Castilla-La Mancha y Extremadura también lo aumentan, un cuarto de punto porcentual.

TABLA 6. Evolución del tipo impositivo aplicado a los Actos JURídicos Documentados POR CC.AA, MODALIDAD ACTO NOTARIAL (CONSTITUCIÓN DE UNA HIPOTECA) (2009-2019) (PORCENTAJES)

\begin{tabular}{|c|c|c|c|c|c|c|c|c|c|c|c|}
\hline $\begin{array}{l}\text { Comunidad } \\
\text { autónoma }\end{array}$ & 2009 & 2010 & 2011 & 2012 & 2013 & 2014 & 2015 & 2016 & 2017 & 2018 & 2019 \\
\hline ESTATAL & $0,50 \%$ & $0,50 \%$ & $0,50 \%$ & $0,50 \%$ & $0,50 \%$ & $0,50 \%$ & $0,50 \%$ & $0,50 \%$ & $0,50 \%$ & $0,50 \%$ & $0,50 \%$ \\
\hline Andalucía & $1,00 \%$ & $1,00 \%$ & $1,00 \%$ & $1,20 \%$ & $1,50 \%$ & $1,50 \%$ & $1,50 \%$ & $1,50 \%$ & $1,50 \%$ & $1,50 \%$ & $1,50 \%$ \\
\hline Aragón & $1,00 \%$ & $1,00 \%$ & $1,00 \%$ & $1,00 \%$ & $1,00 \%$ & $1,00 \%$ & $1,00 \%$ & $1,50 \%$ & $1,50 \%$ & $1,50 \%$ & $1,50 \%$ \\
\hline P. de Asturias & $1,00 \%$ & $1,00 \%$ & $1,20 \%$ & $1,20 \%$ & $1,20 \%$ & $1,20 \%$ & $1,20 \%$ & $1,20 \%$ & $1,20 \%$ & $1,20 \%$ & $1,20 \%$ \\
\hline I. Baleares & $1,00 \%$ & $1,00 \%$ & $1,00 \%$ & $1,00 \%$ & $1,20 \%$ & $1,20 \%$ & $1,20 \%$ & $1,20 \%$ & $1,20 \%$ & $1,20 \%$ & $1,20 \%$ \\
\hline Canarias & $0,75 \%$ & $0,75 \%$ & $0,75 \%$ & $0,75 \%$ & $0,75 \%$ & $0,75 \%$ & $0,75 \%$ & $0,75 \%$ & $0,75 \%$ & $0,75 \%$ & $0,75 \%$ \\
\hline Cantabria & $1,00 \%$ & $1,00 \%$ & $1,00 \%$ & $1,00 \%$ & $1,50 \%$ & $1,50 \%$ & $1,50 \%$ & $1,50 \%$ & $1,50 \%$ & $1,50 \%$ & $1,50 \%$ \\
\hline Castilla y León & $1,00 \%$ & $1,00 \%$ & $1,00 \%$ & $1,00 \%$ & $1,50 \%$ & $1,50 \%$ & $1,50 \%$ & $1,50 \%$ & $1,50 \%$ & $1,50 \%$ & $1,50 \%$ \\
\hline Castilla-La Mancha & $1,00 \%$ & $1,00 \%$ & $1,00 \%$ & $1,00 \%$ & $1,25 \%$ & $1,25 \%$ & $1,25 \%$ & $1,25 \%$ & $1,50 \%$ & $1,50 \%$ & $1,50 \%$ \\
\hline Cataluña & $1,00 \%$ & $1,00 \%$ & $1,20 \%$ & $1,20 \%$ & $1,50 \%$ & $1,50 \%$ & $1,50 \%$ & $1,50 \%$ & $1,50 \%$ & $1,50 \%$ & $1,50 \%$ \\
\hline Extremadura & $1,00 \%$ & $1,00 \%$ & $1,00 \%$ & $1,15 \%$ & $1,20 \%$ & $1,20 \%$ & $1,20 \%$ & $1,20 \%$ & $1,50 \%$ & $1,50 \%$ & $1,50 \%$ \\
\hline Galicia & $1,00 \%$ & $1,00 \%$ & $1,00 \%$ & $1,00 \%$ & $1,50 \%$ & $1,50 \%$ & $1,50 \%$ & $1,50 \%$ & $1,50 \%$ & $1,50 \%$ & $1,50 \%$ \\
\hline C. de Madrid & $1,00 \%$ & $1,00 \%$ & $1,00 \%$ & $1,00 \%$ & $1,00 \%$ & $0,75 \%$ & $0,75 \%$ & $0,75 \%$ & $0,75 \%$ & $0,75 \%$ & $0,75 \%$ \\
\hline R. de Murcia & $1,00 \%$ & $1,00 \%$ & $1,00 \%$ & $1,00 \%$ & $1,50 \%$ & $1,50 \%$ & $1,50 \%$ & $1,50 \%$ & $1,50 \%$ & $1,50 \%$ & $1,50 \%$ \\
\hline La Rioja & $1,00 \%$ & $1,00 \%$ & $1,00 \%$ & $1,00 \%$ & $1,00 \%$ & $1,00 \%$ & $1,00 \%$ & $1,00 \%$ & $1,00 \%$ & $1,00 \%$ & $1,00 \%$ \\
\hline C. Valenciana & $1,00 \%$ & $1,00 \%$ & $1,00 \%$ & $1,20 \%$ & $1,20 \%$ & $1,50 \%$ & $1,50 \%$ & $1,50 \%$ & $1,50 \%$ & $1,50 \%$ & $1,50 \%$ \\
\hline
\end{tabular}

Fuente: Elaboración Propia.

En definitiva, se aprecia como en la actualidad los tipos impositivos sobre Actos Jurídicos Documentados no han variado mucho desde el año 2013 , salvo las cinco Comunidades antes mencionadas, variando sus tipos entre el $0,75 \%$ de la Comunidad de Madrid y el 1,50\%, que han establecido diez de las quince CC.AA que son objeto de estudio. Por tanto, se observa como de forma mayoritaria, en las CC.AA se triplica el tipo impositivo estatal. 
REALA. Nueva Época - N. ${ }^{13}$, abril-septiembre 2020 - ISSN: 1989-8975 - DOI: 10.24965/reala.i13.10746 - [Págs. 131-151]

El impuesto sobre transmisiones patrimoniales y actos jurídicos documentados y el ciclo político: incidencia económica desde ... M. ${ }^{a}$ Ángeles Ortega Almón / Teresa Jesús Vílchez Ortiz / Araceli Rojo Gallego-Burin

El hecho de que las CC.AA, en general, no hayan elevado sus tipos del impuesto de AJD desde 2013, puede haberse debido a que el número de transacciones notariales se ha visto disminuido y estancado como consecuencia de la crisis, sin que el tímido repunte de nuestra economía en estos últimos años haya conseguido levantar el mercado hipotecario.

Como se observa en la Tabla 7, nuestro sujeto pasivo debería de pagar $750 €$ por la constitución de su hipoteca de vivienda habitual, si se le aplica el tipo impositivo estatal, sin embargo, ninguna de las CC.AA se ha acogido a este tipo impositivo.

TAbla 7. Deuda Tributaria a pagar por un SUJeto pasivo por Actos Jurídicos Documentados por CC.AA MODALIDAD ACTO NOTARIAL (CONSTITUCIÓN DE UNA HIPOTECA) (2009-2019) (EN EUROS)

\begin{tabular}{|c|c|c|c|c|c|c|c|c|c|c|c|}
\hline $\begin{array}{l}\text { Comunidad } \\
\text { autónoma }\end{array}$ & 2009 & 2010 & 2011 & 2012 & 2013 & 2014 & 2015 & 2016 & 2017 & 2018 & 2019 \\
\hline ESTATAL & 750 & 750 & 750 & 750 & 750 & 750 & 750 & 750 & 750 & 750 & 750 \\
\hline Andalucía & 1.500 & 1.500 & 1.500 & 1.800 & 2.250 & 2.250 & 2.250 & 2.250 & 2.250 & 2.250 & 2.250 \\
\hline Aragón & 1.500 & 1.500 & 1.500 & 1.500 & 1.500 & 1.500 & 1.500 & 2.250 & 2.250 & 2.250 & 2.250 \\
\hline P. de Asturias & 1.500 & 1.500 & 1.800 & 1.800 & 1.800 & 1.800 & 1.800 & 1.800 & 1.800 & 1.800 & 1.800 \\
\hline I. Baleares & 1.500 & 1.500 & 1.500 & 1.500 & 1.800 & 1.800 & 1.800 & 1.800 & 1.800 & 1.800 & 1.800 \\
\hline Canarias & 1.125 & 1.125 & 1.125 & 1.125 & 1.125 & 1.125 & 1.125 & 1.125 & 1.125 & 1.125 & 1.125 \\
\hline Cantabria & 1.500 & 1.500 & 1.500 & 1.500 & 2.250 & 2.250 & 2.250 & 2.250 & 2.250 & 2.250 & 2.250 \\
\hline Castilla y León & 1.500 & 1.500 & 1.500 & 1.500 & 2.250 & 2.250 & 2.250 & 2.250 & 2.250 & 2.250 & 2.250 \\
\hline Castilla-La Mancha & 1.500 & 1.500 & 1.500 & 1.500 & 1.875 & 1.875 & 1.875 & 1.875 & 2.250 & 2.250 & 2.250 \\
\hline Cataluña & 1.500 & 1.500 & 1.800 & 1.800 & 2.250 & 2.250 & 2.250 & 2.250 & 2.250 & 2.250 & 2.250 \\
\hline Extremadura & 1.500 & 1.500 & 1.500 & 1.725 & 1.800 & 1.800 & 1.800 & 1.800 & 2.250 & 2.250 & 2.250 \\
\hline Galicia & 1.500 & 1.500 & 1.500 & 1.500 & 2.250 & 2.250 & 2.250 & 2.250 & 2.250 & 2.250 & 2.250 \\
\hline C. de Madrid & 1.500 & 1.500 & 1.500 & 1.500 & 1.500 & 1.125 & 1.125 & 1.125 & 1.125 & 1.125 & 1.125 \\
\hline R. de Murcia & 1.500 & 1.500 & 1.500 & 1.500 & 2.250 & 2.250 & 2.250 & 2.250 & 2.250 & 2.250 & 2.250 \\
\hline La Rioja & 1.500 & 1.500 & 1.500 & 1.500 & 1.500 & 1.500 & 1.500 & 1.500 & 1.500 & 1.500 & 1.500 \\
\hline C. Valenciana & 1.500 & 1.500 & 1.500 & 1.800 & 1.800 & 2.250 & 2.250 & 2.250 & 2.250 & 2.250 & 2.250 \\
\hline
\end{tabular}

Fuente: Elaboración Propia.

Como se ha puesto de manifiesto con anterioridad, a día de hoy, existe una gran diferencia entre el impuesto a pagar en la Comunidad de Madrid y Canarias con respecto al resto de Comunidades, aumentando en un $50 \%$ su importe en las diez Comunidades que mantienen el tipo impositivo de AJD más alto. Así en la Comunidad de Madrid, un sujeto pasivo pagaría la cantidad de 1.125€ por la constitución de una hipoteca, para la compra de su vivienda habitual por una cuantía de $150.000 €$. Sin embargo, en otras diez Comunidades tendría que pagar $2.250 €$, por el mismo hecho imponible en concepto de AJD. Con estos datos, se vuelve a constatar las diferencias que supone tributar, por un mismo hecho imponible, en las distintas CC.AA.

En la Figura 3 se puede apreciar más claramente las diferencias que se dan a la hora de tributar, por el mismo hecho imponible, en Comunidades como Madrid, Canarias o La Rioja, respecto de otras, como Andalucía o Comunidad Valenciana. 
Figura 3. Distribución de cuotas a pagar por CC.AA en la modalidad de Actos Jurídicos Documentados POR CONSTITUCIÓN DE HIPOTECA (AÑo 2019) (EN EUROS)

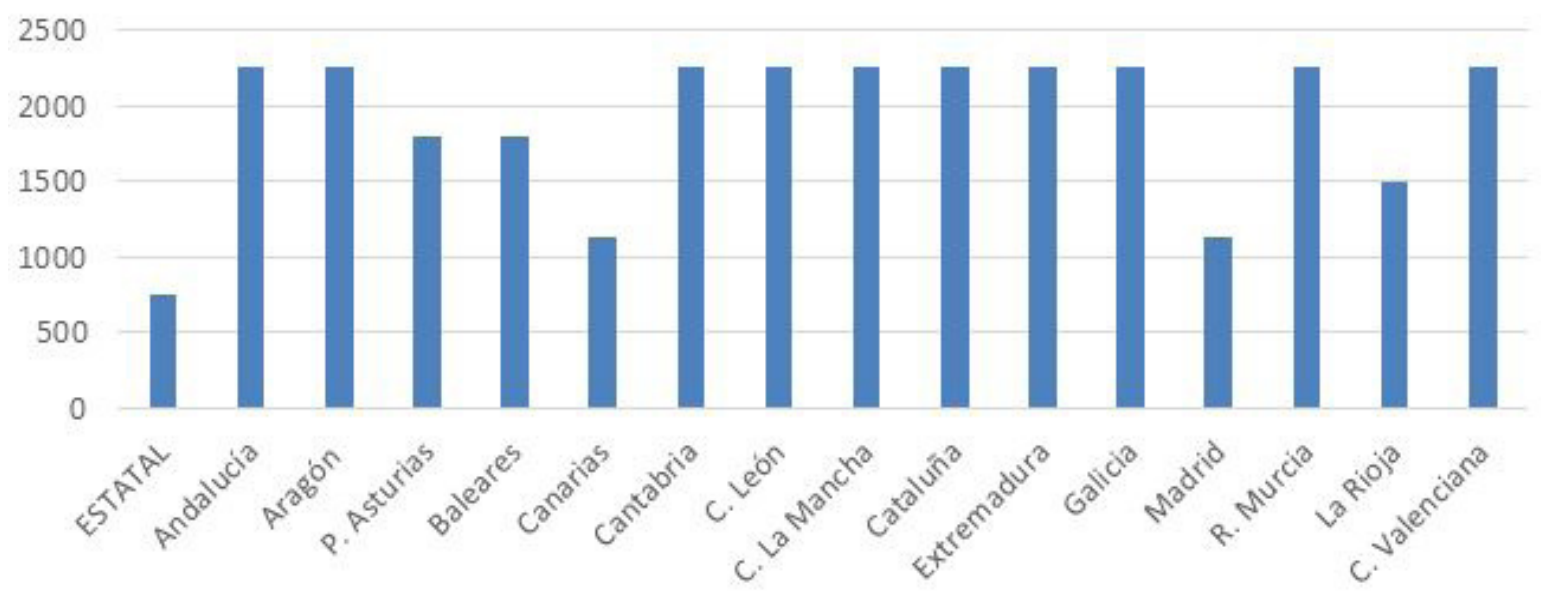

Fuente: Elaboración Propia.

\section{EL ITPAJD Y EL CICLO POLÍTICO EN LAS CC.AA DE RÉGIMEN COMÚN}

La fuerte crisis económica de los últimos años y consiguiente caída de la recaudación, unido a la necesidad de cumplir el objetivo de déficit ha llevado, a algunas CC.AA, a recuperar o incrementar los impuestos cedidos por el Estado, en los que tienen competencias normativas, muy unidos estos cambios, en ambos casos, al ciclo político. Con el objetivo de analizar la posible influencia de éste en la deuda tributaria de un mismo sujeto pasivo en la modalidad de TPO y AJD, se contrastará el análisis realizado, en el epígrafe anterior, sobre la evolución de las diferencias de la deuda tributaria de un mismo sujeto pasivo según la Comunidad, con la evolución de ésta, durante los periodos pre-electorales y post-electorales, en función de la configuración política del Parlamento de cada Comunidad Autónoma. Para alcanzar dicho objetivo, se hará uso de la información suministrada por la Junta Electoral Central sobre la composición de los Parlamentos Autonómicos tras las elecciones, en las dos últimas convocatorias autonómicas. El detalle de la composición de los Parlamentos de la CC.AA se recoge en el Anexo I.

En la Tabla 8, se muestran los tipos impositivos que se aplican desde el año 2009 al 2019 en las CC.AA de régimen común, en la modalidad de Transmisiones Patrimoniales Onerosas de Inmuebles, ya que es esta modalidad la que tiene una mayor incidencia recaudatoria de entre las que lo conforman.

En el Principado de Asturias, Foro Asturias Ciudadanos llega al gobierno en 2011 y sube el tipo de gravamen al $8 \%$, previendo una legislatura completa. Sin embargo, dura solo unos meses, ya que el PSOE toma el relevo en el gobierno a partir de 2012, pactando con IU (Izquierda Unida) y UPyD (Unión Progreso y Democracia), manteniendo el mismo tipo impositivo durante la legislatura.

En Canarias, el partido Coalición Canaria permanece en el gobierno todos los años analizados, mediante pactos con el PSOE y diferentes partidos. Es en esta Comunidad donde se mantiene el tipo más reducido de todas las CC.AA (6,5\%) a excepción de la Comunidad de Madrid (6\%).

TABLA 8. EVolución del tipo impositivo aplicado a las Transmisiones Patrimoniales de Inmuebles POR CC.AA (2009-2019) (PORCENTAJES)

\begin{tabular}{lrrrrrrrrrrr}
\hline CC.AA/Años & $\mathbf{2 0 0 9}$ & $\mathbf{2 0 1 0}$ & $\mathbf{2 0 1 1}$ & $\mathbf{2 0 1 2}$ & $\mathbf{2 0 1 3}$ & $\mathbf{2 0 1 4}$ & $\mathbf{2 0 1 5}$ & $\mathbf{2 0 1 6}$ & $\mathbf{2 0 1 7}$ & $\mathbf{2 0 1 8}$ & $\mathbf{2 0 1 9}$ \\
\hline Estatal & $6 \%$ & $6 \%$ & $6 \%$ & $6 \%$ & $6 \%$ & $6 \%$ & $6 \%$ & $6 \%$ & $6 \%$ & $6 \%$ & $6 \%$ \\
\hline Andalucía & $7 \%$ & $7 \%$ & $7 \%$ & $\mathbf{8} \%$ & $8 \%$ & $8 \%$ & $8 \%$ & $8 \%$ & $8 \%$ & $8 \%$ & $8 \%$ \\
\hline Aragón & $7 \%$ & $7 \%$ & $7 \%$ & $7 \%$ & $7 \%$ & $7 \%$ & $7 \%$ & $8 \%$ & $8 \%$ & $8 \%$ & $8 \%$ \\
\hline
\end{tabular}


REALA. Nueva Época - N. ${ }^{13}$, abril-septiembre 2020 - ISSN: 1989-8975 - DOI: 10.24965/reala.i13.10746 - [Págs. 131-151]

El impuesto sobre transmisiones patrimoniales y actos jurídicos documentados y el ciclo político: incidencia económica desde ... M. ${ }^{a}$ Ángeles Ortega Almón / Teresa Jesús Vílchez Ortiz / Araceli Rojo Gallego-Burin

\begin{tabular}{lrrrrrrrrrrrr}
\hline CC.AA/Años & $\mathbf{2 0 0 9}$ & $\mathbf{2 0 1 0}$ & $\mathbf{2 0 1 1}$ & $\mathbf{2 0 1 2}$ & $\mathbf{2 0 1 3}$ & $\mathbf{2 0 1 4}$ & $\mathbf{2 0 1 5}$ & $\mathbf{2 0 1 6}$ & $\mathbf{2 0 1 7}$ & $\mathbf{2 0 1 8}$ & $\mathbf{2 0 1 9}$ \\
\hline P. de Asturias & $7 \%$ & $7 \%$ & $\mathbf{8} \%$ & $8 / \%$ & $8 \%$ & $8 \%$ & $8 \%$ & $8 \%$ & $8 \%$ & $8 \%$ & $8 \%$ \\
\hline I. Baleares & $7 \%$ & $7 \%$ & $7 \%$ & $7 \%$ & $\mathbf{8} \%$ & $8 \%$ & $8 \%$ & $8 \%$ & $8 \%$ & $8 \%$ & $8 \%$ \\
\hline Canarias & $6,50 \%$ & $6,50 \%$ & $6,50 \%$ & $6,50 \%$ & $6,50 \%$ & $6,50 \%$ & $6,50 \%$ & $6,50 \%$ & $6,50 \%$ & $6,50 \%$ & $6,50 \%$ \\
\hline Cantabria & $7 \%$ & $7 \%$ & $7 \%$ & $7 \%$ & $\mathbf{8} \%$ & $8 \%$ & $8 \%$ & $8 \%$ & $8 \%$ & $10 \%$ & $9 \%$ \\
\hline Castilla y León & $7 \%$ & $7 \%$ & $7 \%$ & $7 \%$ & $8 \%$ & $8 \%$ & $8 \%$ & $8 \%$ & $8 \%$ & $8 \%$ & $8 \%$ \\
\hline Castilla-La Mancha & $7 \%$ & $7 \%$ & $7 \%$ & $7 \%$ & $8 \%$ & $8 \%$ & $8 \%$ & $8 \%$ & $\mathbf{9} \%$ & $9 \%$ & $9 \%$ \\
\hline Cataluña & $7 \%$ & $7 \%$ & $\mathbf{8} \%$ & $8 \%$ & $8 \%$ & $10 \%$ & $10 \%$ & $10 \%$ & $10 \%$ & $10 \%$ & $10 \%$ \\
\hline Extremadura & $7 \%$ & $7 \%$ & $7 \%$ & $7 \%$ & $\mathbf{8} \%$ & $8 \%$ & $8 \%$ & $8 \%$ & $8 \%$ & $8 \%$ & $8 \%$ \\
\hline Galicia & $7 \%$ & $7 \%$ & $7 \%$ & $7 \%$ & $10 \%$ & $10 \%$ & $10 \%$ & $10 \%$ & $10 \%$ & $10 \%$ & $10 \%$ \\
\hline C. de Madrid & $7 \%$ & $7 \%$ & $7 \%$ & $7 \%$ & $7 \%$ & $6 \%$ & $6 \%$ & $6 \%$ & $6 \%$ & $6 \%$ & $6 \%$ \\
\hline R. de Murcia & $7 \%$ & $7 \%$ & $7 \%$ & $7 \%$ & $8 \%$ & $8 \%$ & $8 \%$ & $8 \%$ & $8 \%$ & $8 \%$ & $8 \%$ \\
\hline La Rioja & $7 \%$ & $7 \%$ & $7 \%$ & $7 \%$ & $7 \%$ & $7 \%$ & $7 \%$ & $7 \%$ & $7 \%$ & $7 \%$ & $7 \%$ \\
\hline C. Valenciana & $7 \%$ & $7 \%$ & $7 \%$ & $7 \%$ & $8 \%$ & $10 \%$ & $10 \%$ & $10 \%$ & $10 \%$ & $10 \%$ & $10 \%$ \\
\hline
\end{tabular}

Fuente: Elaboración Propia.

En Cataluña, CiU (Convergencia y Unión) gana las elecciones con mayoría simple en 2010 y ya en periodo post-electoral (2011) sube el impuesto. Obligada a convocar elecciones, por la inestabilidad política, en noviembre de 2012, revalida su gobierno pero en coalición con ERC (Esquerra Republicana de Cataluña), de cuyo pacto proviene la iniciativa de subir el impuesto en 2014 a un 10\%. Se convocan elecciones en 2015 en las que toma el relevo JxSí (Junts per Sí) pactando con la CUP (Candidatura d'Unitat Popular-Alternativa d'Esquerres) y mantienen el tipo impositivo sin cambios, dada la dificultosa situación política que atraviesan en los siguientes años.

En el caso de Andalucía, el PSOE gana las elecciones en marzo de 2012 pactando con IU. Ese mismo año, y como consecuencia del pacto acuerdan una subida post-electoral del tipo impositivo al $8 \%$, el cual se ha mantenido sin cambios pues dicho partido ha seguido gobernando hasta 2018 , aunque con diferentes socios de gobierno y de distinto signo político (IU, CIUDADANOS).

Las Comunidades de Islas Baleares, Castilla y León, Extremadura y Región de Murcia, con gobiernos del PP desde 2011, suben el impuesto del $7 \%$ al $8 \%$, dos años después de su entrada en el gobierno (en 2013) manteniéndolo hasta la actualidad. Galicia gobernada por el PP con mayoría absoluta, al igual que las anteriores comunidades sube su impuesto al $10 \%$ en el mismo año, manteniéndolo también hasta la actualidad. Esta subida de 2013 en Comunidades regidas por el PP se debieron tomar por la urgente necesidad de incrementar la recaudación que hasta ese momento había sido muy baja causada por la crisis y el estallido de la burbuja inmobiliaria, y teniendo en cuenta que ya en dicho año se tenían indicadores de una tímida recuperación.

En 2011 en Aragón gobierna el PP mediante pacto con el PAR (Partido Aragonesista). Estos deciden mantener un tipo impositivo bajo del $7 \%$ hasta que en las elecciones de 2015 se produce un cambio de gobierno donde el PSOE pacta con PODEMOS, CHA (Chunta Aragonesista) e IU y es en el periodo postelectoral (año 2016) cuando suben el impuesto al $8 \%$, manteniéndolo hasta la actualidad.

En las Comunidades de Cantabria y Castilla-La Mancha el PP, después de dos años de gobierno, en 2013, sube el impuesto al $8 \%$. En las elecciones de 2015, en Castilla-La Mancha gobierna el PSOE por pacto con PODEMOS y en ese mismo año, en Cantabria gobierna el PRC (Partido Regionalista de Cantabria) por pacto con el PSOE. En ambos casos van a realizar una subida del tipo impositivo al $9 \%$ y al $10 \%$, en los años 2017 y 2018 respectivamente. En el caso de Cantabria, en 2019 lo bajará al 9\%, coincidiendo con un año electoral y probablemente como medida preelectoral.

Es de destacar que Comunidades como Madrid y La Rioja gobernadas por el PP con mayorías absolutas o mediante pactos con CIUDADANOS hayan mantenido tipos impositivos bajos (6\% y $7 \%$ ) e incluso llegando a rebajarlo al $6 \%$ en el 2014, en el caso de la Comunidad de Madrid, coincidiendo con la cercanía de las elecciones de 2015. 
REALA. Nueva Época - N. ${ }^{13}$, abril-septiembre 2020 - ISSN: 1989-8975 - DOI: 10.24965/reala.i13.10746 - [Págs. 131-151]

El impuesto sobre transmisiones patrimoniales y actos jurídicos documentados y el ciclo político: incidencia económica desde ... M. ${ }^{a}$ Ángeles Ortega Almón / Teresa Jesús Vílchez Ortiz / Araceli Rojo Gallego-Burin

En la Comunidad Valenciana el PP, dos años después de estar en el gobierno, sube el impuesto de forma sucesiva en los años 2013 y 2014 hasta llevarlo al 10\%, el cual será mantenido por el gobierno del PSOE, PODEMOS y COMPROMIS desde 2015 en adelante.

En la Tabla 9 se muestran los tipos impositivos entre los años 2009 y 2019 sobre el Impuesto de Actos Jurídicos Documentados en la modalidad de Acto Notarial por constitución de una hipoteca.

De acuerdo con las tablas del Anexo I, en la Comunidad de Andalucía en las elecciones de marzo de 2012 el PSOE, pactando con IU, consigue continuar en el gobierno. En ese mismo año y el siguiente (etapa post-electoral) van a subir el IAJD hasta alcanzar el 1,50\% en 2013. Para la siguiente convocatoria de elecciones en 2015, en las que sigue gobernando el PSOE pero mediante pacto con CIUDADANOS, deciden mantenerlo hasta la actualidad.

En la Comunidad de Aragón se repiten las mismas circunstancias que en el ITPO para el IAJD pero la subida es del $1 \%$ al $1,50 \%$, y en el $P$. de Asturias ocurre algo muy similar pero la subida será del $1 \%$ al $1,20 \%$. Las Comunidades de Islas Baleares, Cantabria, Castilla y León, Galicia y Región de Murcia con gobiernos del PP desde 2011, deciden subir el IAJD en el 2013 (medida post-electoral) al 1,5\%, salvo Islas Baleares que lo sube al 1,20\%. Dicha subida puede tener similar interpretación que la subida del ITPO, pues en ambos casos se ven en la necesidad de incrementar los ingresos tributarios, tras haber sufrido la parte más complicada de la crisis.

En las Islas Canarias ocurre algo muy similar a lo que sucede con el ITPO, esta Comunidad mantiene el tipo más bajo del impuesto de todas las comunidades durante todos estos años $(0,75 \%)$, ya que sigue en el gobierno el mismo partido COALICIÓN CANARIA.

En el caso de Castilla-La Mancha sucede con el IAJD igual que con el ITPO, en el año 2016 el PP sube el impuesto al 1,25\% dos años después a su llegada al gobierno, y el PSOE junto con Podemos también lo suben en el 2017 (subida post-electoral).

TABLA 9. Evolución del tipo impositivo aplicado a los ACtos JuRídicos Documentados por CC.AA, MODALIDAD ACTO NOTARIAL (CONSTITUCIÓN DE UNA HIPOTECA) (2009-2019) (PORCENTAJES)

\begin{tabular}{|c|c|c|c|c|c|c|c|c|c|c|c|}
\hline $\begin{array}{l}\text { Comunidad } \\
\text { autónoma }\end{array}$ & 2009 & 2010 & 2011 & 2012 & 2013 & 2014 & 2015 & 2016 & 2017 & 2018 & 2019 \\
\hline ESTATAL & $0,50 \%$ & $0,50 \%$ & $0,50 \%$ & $0,50 \%$ & $0,50 \%$ & $0,50 \%$ & $0,50 \%$ & $0,50 \%$ & $0,50 \%$ & $0,50 \%$ & $0,50 \%$ \\
\hline Andalucía & $1,00 \%$ & $1,00 \%$ & $1,00 \%$ & $1,20 \%$ & $1,50 \%$ & $1,50 \%$ & $1,50 \%$ & $1,50 \%$ & $1,50 \%$ & $1,50 \%$ & $1,50 \%$ \\
\hline Aragón & $1,00 \%$ & $1,00 \%$ & $1,00 \%$ & $1,00 \%$ & $1,00 \%$ & $1,00 \%$ & $1,00 \%$ & $1,50 \%$ & $1,50 \%$ & $1,50 \%$ & $1,50 \%$ \\
\hline P. de Asturias & $1,00 \%$ & $1,00 \%$ & $1,20 \%$ & $1,20 \%$ & $1,20 \%$ & $1,20 \%$ & $1,20 \%$ & $1,20 \%$ & $1,20 \%$ & $1,20 \%$ & $1,20 \%$ \\
\hline I. Baleares & $1,00 \%$ & $1,00 \%$ & $1,00 \%$ & $1,00 \%$ & $1,20 \%$ & $1,20 \%$ & $1,20 \%$ & $1,20 \%$ & $1,20 \%$ & $1,20 \%$ & $1,20 \%$ \\
\hline Canarias & $0,75 \%$ & $0,75 \%$ & $0,75 \%$ & $0,75 \%$ & $0,75 \%$ & $0,75 \%$ & $0,75 \%$ & $0,75 \%$ & $0,75 \%$ & $0,75 \%$ & $0,75 \%$ \\
\hline Cantabria & $1,00 \%$ & $1,00 \%$ & $1,00 \%$ & $1,00 \%$ & $1,50 \%$ & $1,50 \%$ & $1,50 \%$ & $1,50 \%$ & $1,50 \%$ & $1,50 \%$ & $1,50 \%$ \\
\hline Castilla y León & $1,00 \%$ & $1,00 \%$ & $1,00 \%$ & $1,00 \%$ & $1,50 \%$ & $1,50 \%$ & $1,50 \%$ & $1,50 \%$ & $1,50 \%$ & $1,50 \%$ & $1,50 \%$ \\
\hline Castilla-La Mancha & $1,00 \%$ & $1,00 \%$ & $1,00 \%$ & $1,00 \%$ & $1,25 \%$ & $1,25 \%$ & $1,25 \%$ & $1,25 \%$ & $1,50 \%$ & $1,50 \%$ & $1,50 \%$ \\
\hline Cataluña & $1,00 \%$ & $1,00 \%$ & $1,20 \%$ & $1,20 \%$ & $1,50 \%$ & $1,50 \%$ & $1,50 \%$ & $1,50 \%$ & $1,50 \%$ & $1,50 \%$ & $1,50 \%$ \\
\hline Extremadura & $1,00 \%$ & $1,00 \%$ & $1,00 \%$ & $1,15 \%$ & $1,20 \%$ & $1,20 \%$ & $1,20 \%$ & $1,20 \%$ & $1,50 \%$ & $1,50 \%$ & $1,50 \%$ \\
\hline Galicia & $1,00 \%$ & $1,00 \%$ & $1,00 \%$ & $1,00 \%$ & $1,50 \%$ & $1,50 \%$ & $1,50 \%$ & $1,50 \%$ & $1,50 \%$ & $1,50 \%$ & $1,50 \%$ \\
\hline C. de Madrid & $1,00 \%$ & $1,00 \%$ & $1,00 \%$ & $1,00 \%$ & $1,00 \%$ & $0,75 \%$ & $0,75 \%$ & $0,75 \%$ & $0,75 \%$ & $0,75 \%$ & $0,75 \%$ \\
\hline R. de Murcia & $1,00 \%$ & $1,00 \%$ & $1,00 \%$ & $1,00 \%$ & $1,50 \%$ & $1,50 \%$ & $1,50 \%$ & $1,50 \%$ & $1,50 \%$ & $1,50 \%$ & $1,50 \%$ \\
\hline La Rioja & $1,00 \%$ & $1,00 \%$ & $1,00 \%$ & $1,00 \%$ & $1,00 \%$ & $1,00 \%$ & $1,00 \%$ & $1,00 \%$ & $1,00 \%$ & $1,00 \%$ & $1,00 \%$ \\
\hline C. Valenciana & $1,00 \%$ & $1,00 \%$ & $1,00 \%$ & $1,20 \%$ & $1,20 \%$ & $1,50 \%$ & $1,50 \%$ & $1,50 \%$ & $1,50 \%$ & $1,50 \%$ & $1,50 \%$ \\
\hline
\end{tabular}

Fuente: Elaboración Propia. 
En Cataluña se repite el mismo comportamiento que con el ITPO, CyU sube en 2011 el impuesto a 1,2\% después de estar un año en el gobierno. Y después de las elecciones del 2012, en el año 2013 habiendo pactado con ERC vuelve a subirlo a 1,50\% para mantenerlo hasta la actualidad.

En el caso de Extremadura el gobierno del PP subirá el impuesto en los años 2012 y 2013 para llevarlo hasta el 1,20\%. Pero después de que el PSOE gane las elecciones en 2015 este lo volverá a subir en 2017 al $1,50 \%$, seguramente como consecuencia de las medidas pactadas con PODEMOS.

En la Comunidad de Madrid, al igual que ocurre con el ITPO, gobierna desde 2011 el PP con mayoría absoluta, el cual mantiene un tipo impositivo del $1 \%$ hasta que en el 2014 , previendo las elecciones de 2015 y como medida preelectoral de propaganda, lo baja aún más $(0,75 \%)$. Algo similar le ocurre a la $C$. de La Rioja, la cual estando gobernada en todos estos años por el PP con mayorías absoluta y simple, no ha variado su tipo impositivo del $1 \%$ en todos los años estudiados.

La Comunidad Valenciana, repitiendo el patrón del ITPO, va a ver incrementado su IAJD por el PP en dos ocasiones, en los años 2012 y 2014 hasta llevarlo al 1,50\%. Posteriormente en el 2015 el PSOE junto con COMPROMÍS y PODEMOS dejará el mismo tipo impositivo.

\section{CONCLUSIONES}

La imposición sobre la riqueza es una herramienta fundamental en todo país desarrollado, que pretenda fundamentar su política fiscal sobre la base de la equidad y el derecho a la igualdad de oportunidades de todos sus ciudadanos. Contribuye a lograr una mejor redistribución de la riqueza, incentiva una utilización más productiva de los bienes y permite un mayor control recaudatorio, procurando una disminución del fraude fiscal.

En nuestro país, los impuestos que gravan la riqueza y cuya recaudación total está cedida a las CC.AA de régimen común, contando con capacidad normativa y de gestión de éstos, son: el Impuesto sobre el Patrimonio Neto (IPN), el Impuesto de Sucesiones y Donaciones (ISD) y el Impuesto de Transmisiones Patrimoniales y Actos Jurídicos Documentados (ITPAJD).

Este trabajo se centra en el estudio del ITPAJD, en sus dos modalidades de Transmisiones Patrimoniales Onerosas (TPO) y Actos Jurídicos Documentados (AJD), del cual las CC.AA tienen cedidas las capacidades normativas para modificar los elementos básicos y determinantes, como son los mínimos exentos, tipos impositivos, deducciones y bonificaciones, los cuales determinan la progresividad efectiva de éstos. Ello implica que un ciudadano por un mismo hecho imponible tenga que afrontar una deuda tributaria diferente según la Comunidad Autónoma. En la modalidad de TPO, la mayoría de las CC.AA han optado por aplicar tipos impositivos por encima del establecido a nivel estatal, creando a su vez tipos reducidos para determinadas clases de hechos imponibles (asociados, en su mayoría, a determinadas situaciones sociales de vulnerabilidad). En cuanto a este hecho se aprecia que los colectivos más beneficiados para la adquisición de vivienda habitual, en la mayoría de las CC.AA son los jóvenes, las familias numerosas y los colectivos con minusvalías. Además existen dos nuevos colectivos que se comienzan a tener en cuenta en estos beneficios, como son las víctimas de violencia de género y las familias monoparentales. Entre las Comunidades con los tipos impositivos más bajos, destaca la Comunidad de Madrid, igualándose al tipo estatal $(6 \%)$, lo que sin lugar a dudas incentiva una competencia fiscal entre las CC.AA. En esta modalidad, la mayoría de las CC.AA eligen un tipo de gravamen proporcional, aunque se observa una tendencia cada vez mayor a utilizar el progresivo, en distintos tramos, según sea el valor del inmueble, lo que se acerca más a una imposición equitativa y adecuada al valor real del hecho imponible.

En cuanto a la modalidad de AJD, se aprecia cómo la mayoría han establecido un tipo de gravamen que triplica al tipo estatal $(1,50 \%)$, sin que ninguna lo iguale, siendo otra vez la Comunidad de Madrid una de las que tiene un tipo más bajo $(0,75 \%)$.

Al analizar la incidencia presupuestaria del ITPAJD se observa que su peso contributivo en el año 2017, en el conjunto de las CC.AA, ha sido fundamental respecto del total de los tributos cedidos no sujetos a liquidación, comportando más de la mitad de la recaudación obtenida por estos impuestos. Se debe, sobre todo, a la recaudación del ITPO, cuya aportación ha sido mayoritaria respecto al resto de los impuestos. Sin embargo, su contribución respecto a la recaudación tributaria total queda muy mermada, constituyendo sólo el $8,59 \%$ respecto de dicha recaudación (pues es aquí donde adquieren mayor relevancia los impuestos sujetos a liquidación).

Valorando su evolución en el periodo 2006-2016, este impuesto en sus dos modalidades (ITP y AJD) sufre un descenso durante los años previos a la crisis, llegando a tocar fondo en el 2011 y 2009 respectiva- 
mente, para mantener dichos niveles el resto del periodo estudiado. Sin embargo, la recaudación total sigue distinto camino, experimentando subidas en 2009 y continúa en ascenso todo el resto del periodo. Dicho ascenso fue potenciado por dos factores claves, como fueron la Reforma del Sistema de Financiación de las CC.AA en 2009 y las subidas de los tipos impositivos del IVA en 2012.

Al analizar la incidencia económica del ITPAJD sobre el sujeto pasivo, observando la evolución de los tipos impositivos aplicados por las distintas CC.AA de régimen común, en las modalidades de TPO y AJD, se ha comprobado la existencia de notables desigualdades para los ciudadanos, a la hora de tributar por un mismo hecho imponible. Su causa principal se haya, en la capacidad normativa que dichas CC.AA tienen sobre estos impuestos, pudiendo así aplicar tipos de gravámenes, reducciones, deducciones y bonificaciones bajo las condiciones que le sean más convenientes, a los intereses de las políticas fiscales de los gobiernos autonómicos, según la configuración política del Parlamento de cada Comunidad. Se observa cómo, en la modalidad de TPO, en el supuesto analizado, en el año 2009 apenas existían diferencias apreciables entre las Comunidades. Actualmente, existen diferencias en los tipos de gravámenes de hasta cuatro puntos porcentuales, significando cada punto, un aumento a pagar en la cuota tributaria de $1.500 €$.

En la modalidad de AJD, se siguen apreciando notables desigualdades, que si bien son menos gravosas para la ciudadanía, siguen poniendo de manifiesto, el diferente trato fiscal para un mismo sujeto pasivo, por un mismo hecho imponible, según la Comunidad. Como se ha señalado anteriormente, en diez de las quince CC.AA se triplica el tipo de gravamen respecto del estatal, además de observar cómo en ciertas CC.AA se tributa la «mitad» que en otras por el mismo hecho imponible (Canarias y Comunidad de Madrid).

Asimismo, en el estudio de la relación entre los cambios políticos y la incidencia tributaria que éstos pueden provocar en las distintas CC.AA, se ha podido constatar, que las subidas o bajadas de los tipos impositivos del ITPAJD, además de estar relacionadas con las necesidades recaudatorias (como hemos visto en el proceder de distintas CC.AA, donde en 2013 optan por una subida de los impuestos), están significativamente relacionadas con los cambios provocados por los ciclos políticos y por los periodos pre 0 post-electorales. Así se aprecia que, cuando la tendencia política del partido gobernante es de signo liberal o conservador, los tipos impositivos bajan o se mantienen bajos. A diferencia de los gobiernos que se definen como de centro-izquierda o de izquierda del espectro político, que suben los tipos impositivos.

Por otro lado, también se observa cómo en periodos preelectorales, los gobiernos de ambos signos del espectro político, tienden a disminuir o mantener dichos tipos impositivos como medidas preelectorales de propaganda. Y si se da un cambio de gobierno, el entrante tendrá la opción de subirlo o mantenerlo en función de los acuerdos y pactos de gobierno que deba o no realizar. También cabe puntualizar que si el gobierno posee mayoría absoluta, no viéndose en la necesidad de acordar con otras formaciones políticas, tiene todo el poder de implementar la política fiscal que sea más afín a su tendencia política.

En último lugar, se ha podido observar que en las Comunidades donde no ha habido cambios de gobierno, éstas han mantenido el mismo tipo impositivo durante todos los años de gobernanza de dichos partidos o coaliciones (La Rioja, Canarias).

En el objeto de intentar paliar, en lo posible, estas desigualdades y la posible competencia fiscal que se produce como consecuencia de ésta, sería recomendable acordar unos límites razonables entre las CC.AA y el Estado de los elementos principales que determinan la deuda. Es positivo que las CC.AA tengan capacidad para subir o bajar algunos impuestos, pero estableciéndose unos mínimos y máximos (horquilla) para todas las Comunidades, así cada una de ellas será corresponsable de su situación financiera, respetando de esta forma los objetivos marcados por Hacienda (controlando el déficit), así como rindiendo cuentas ante sus ciudadanos, por una mala gestión del gasto o por impuestos excesivos. El aumento de la deuda pública autonómica ha convivido en numerosos casos con «políticas tributarias laxas». 
REALA. Nueva Época - N. ${ }^{13}$, abril-septiembre 2020 - ISSN: 1989-8975 - DOI: 10.24965/reala.i13.10746 - [Págs. 131-151]

El impuesto sobre transmisiones patrimoniales y actos jurídicos documentados y el ciclo político: incidencia económica desde ... M. ${ }^{a}$ Ángeles Ortega Almón / Teresa Jesús Vílchez Ortiz / Araceli Rojo Gallego-Burin

ANEXO I. RESULTADOS ELECTORALES DE LAS CC.AA DE RÉGIMEN COMÚN (2011-2018)

\begin{tabular}{|c|c|c|c|c|c|c|c|c|}
\hline \multicolumn{9}{|c|}{ PARLAMENTO DE LA JUNTA DE ANDALUCÍA } \\
\hline \multicolumn{3}{|c|}{$\begin{array}{l}\text { DIPUTADOS ELECCIONES } \\
2012\end{array}$} & \multicolumn{3}{|c|}{$\begin{array}{c}\text { DIPUTADOS ELECCIONES } \\
2015^{\mathrm{a}}\end{array}$} & \multicolumn{3}{|c|}{ DIPUTADOS ELECCIONES 2018 } \\
\hline PSOE, 47 & PP, 50 & $\mathrm{IU}, 12$ & PSOE, 47 & PP, 33 & PODEMOS, 15 & PSOE, 33 & PP, 26 & Cs., 21 \\
\hline & & & Cs. ${ }^{c}, 9$ & $\mathrm{IU}, 5$ & & ADELANTE A. ${ }^{d}, 17$ & VOX, 12 & \\
\hline \multicolumn{3}{|c|}{$\begin{array}{l}\text { Gobierno del PSOE por } \\
\text { pacto con IU }\end{array}$} & \multicolumn{3}{|c|}{$\begin{array}{l}\text { Gobierno del PSOE por pacto } \\
\text { con CIUDADANOS }\end{array}$} & \multicolumn{3}{|c|}{$\begin{array}{l}\text { Gobierno del PP por pacto } \\
\text { con CIUDADANOS }\end{array}$} \\
\hline \multicolumn{9}{|c|}{$\begin{array}{l}\text { a Las elecciones se adelantan un año por dificultades de gobernabilidad con IU. } \\
\text { b Se adelantan las elecciones un año por dificultades de gobernabilidad con CIUDADANOS. } \\
\text { c CIUDADANOS. } \\
\text { d ADELANTE ANDALUCÍA. }\end{array}$} \\
\hline
\end{tabular}

\section{CORTES DE ARAGÓN}

\begin{tabular}{l|c|c|c|c|c}
\hline \multicolumn{2}{c|}{ DIPUTADOS ELECCIONES 2011 } & \multicolumn{2}{c}{ DIPUTADOS ELECCIONES 2015 } \\
\hline PP, 30 & PSOE, 22 & PAR, 7 & PP, 21 & PSOE, 18 & PODEMOS, 14 \\
\hline CHA $^{\mathrm{b}}, 4$ & $\mathrm{IU}, 4$ & & PAR, 6 & $\mathrm{Cs}, 5$ & $\mathrm{CHA}, 2$ \\
\hline & & $\mathrm{IU}, 1$ & & \\
\hline & & Gobierno del PSOE por pacto con PODEMOS, CHA e IU \\
\hline
\end{tabular}

JUNTA GENERAL DEL PRINCIPADO DE ASTURIAS

\begin{tabular}{|c|c|c|c|c|c|c|c|c|}
\hline \multicolumn{3}{|c|}{ DIPUTADOS ELECCIONES 2011} & \multicolumn{3}{|c|}{ DIPUTADOS ELECCIONES 2012 ${ }^{\mathrm{b}}$} & \multicolumn{3}{|c|}{ DIPUTADOS ELECCIONES 2015} \\
\hline $\mathrm{FAC}^{\mathrm{a}}, 16$ & PSOE, 15 & PP, 10 & PSOE, 17 & FAC, 12 & PP, 7 & PSOE, 14 & $\mathrm{PP}, 11$ & $\mathrm{IU}, 5$ \\
\hline $\mathrm{IU}, 4$ & & & $\mathrm{IU}, 5$ & UPyD ${ }^{c}, 1$ & & FAC, 3 & PODEMOS, 9 & Cs, 3 \\
\hline \multicolumn{3}{|c|}{$\begin{array}{l}\text { Gobierno del FAC con mayoría } \\
\text { simple }\end{array}$} & \multicolumn{3}{|c|}{$\begin{array}{l}\text { Gobierno del PSOE por pacto } \\
\text { con IU y UPyD }\end{array}$} & \multicolumn{3}{|c|}{ Gobierno del PSOE por pacto con IU } \\
\hline \multicolumn{9}{|c|}{$\begin{array}{l}\text { a } \text { FORO ASTURIAS CIUDADANOS. } \\
\text { b Se adelantan las elecciones } 3 \text { años, transcurridos } 6 \text { meses de gobierno. } \\
\text { c UNIÓN PROGRESO Y DEMOCRACIA. }\end{array}$} \\
\hline
\end{tabular}

\section{PARLAMENTO DE LAS ISLAS BALEARES}

\begin{tabular}{|c|c|c|c|c|c|}
\hline \multicolumn{3}{|c|}{ DIPUTADOS ELECCIONES 2011} & \multicolumn{3}{|c|}{ DIPUTADOS ELECCIONES 2015} \\
\hline PP, 35 & PSOE, 14 & PSM-IV-EXMa, 4 & PP, 20 & PSIB-PSOE ${ }^{\mathrm{e}}, 14$ & PODEMOS, 10 \\
\hline \multirow[t]{2}{*}{ PSOE-PACTE ${ }^{\mathrm{b}}, 4$} & PSM-EN" ${ }^{c} 1$ & GXF + PSOE ${ }^{d}, 1$ & MÉS, 6 & EL PIfg, 3 & $\mathrm{MpM}^{\mathrm{h}}, 3$ \\
\hline & & & Cs, 2 & GXF+PSIB, 1 & \\
\hline \multicolumn{3}{|c|}{ Gobierno del PP por mayoría absoluta } & \multicolumn{3}{|c|}{$\begin{array}{c}\text { Gobierno del PSOE por pacto con PODEMOS, } \\
\text { MÉS y GXF }\end{array}$} \\
\hline
\end{tabular}

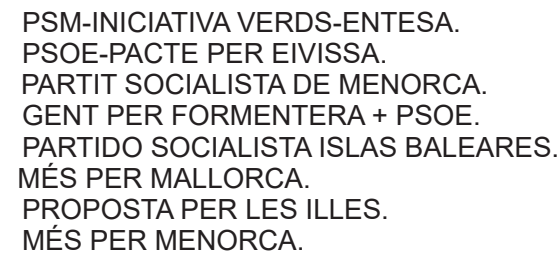


REALA. Nueva Época - N. ${ }^{13}$, abril-septiembre 2020 - ISSN: 1989-8975 - DOI: 10.24965/reala.i13.10746 - [Págs. 131-151]

El impuesto sobre transmisiones patrimoniales y actos jurídicos documentados y el ciclo político: incidencia económica desde ... M. ${ }^{a}$ Ángeles Ortega Almón / Teresa Jesús Vílchez Ortiz / Araceli Rojo Gallego-Burin

\section{PARLAMENTO DE LAS ISLAS CANARIAS}

\begin{tabular}{|c|c|c|c|c|c|}
\hline \multicolumn{3}{|c|}{ DIPUTADOS ELECCIONES 2011} & \multicolumn{3}{|c|}{ DIPUTADOS ELECCIONES 2015} \\
\hline PP, 20 & CC-PNC-CCNa, 21 & PSOE, 15 & CC-PNC-AHÍ ${ }^{b}, 18$ & PSOE, 15 & PP, 12 \\
\hline NUEVA CANARIAS, 3 & & & PODEMOS, 7 & NUEVA CANARIAS, 5 & $\mathrm{ASG}^{\mathrm{c}}, 3$ \\
\hline \multicolumn{3}{|c|}{$\begin{array}{l}\text { Gobierno de Coalición Canaria } \\
\text { por pacto con el PSOE }\end{array}$} & \multicolumn{3}{|c|}{$\begin{array}{c}\text { Gobierno de Coalición Canaria por pacto con } \\
\text { PNC, AHÍ, PSOE y ASG }\end{array}$} \\
\hline \multicolumn{6}{|c|}{$\begin{array}{ll}\text { a } & \text { COALICIÓN CANARIA-PARTIDO NACIONALISTA CANARIO. } \\
\text { b COALICIÓN CANARIA-PARTIDO NACIONALISTA CANARIO-AGRUPACIÓN HERREÑA INDEPENDIENTE. } \\
\text { c } \\
\text { AGRUPACIÓN SOCIALISTA GOMERA. }\end{array}$} \\
\hline \multicolumn{6}{|c|}{ PARLAMENTO DE CANTABRIA } \\
\hline \multicolumn{3}{|c|}{ DIPUTADOS ELECCIONES 2011} & \multicolumn{3}{|c|}{ DIPUTADOS ELECCIONES 2015} \\
\hline \multirow[t]{2}{*}{ PP, 20} & $\mathrm{RC}^{\mathrm{a}}, 12$ & PSOE, 7 & PP, 13 & PRC, 12 & PSOE, 5 \\
\hline & & & PODEMOS, 3 & Cs, 2 & \\
\hline \multicolumn{3}{|c|}{ Gobierno del PP con mayoría absoluta } & \multicolumn{3}{|c|}{ Gobierno del PRC por pacto con el PSOE } \\
\hline
\end{tabular}

CORTES DE CASTILLA LA MANCHA

\begin{tabular}{|c|c|c|c|c|c|}
\hline \multicolumn{3}{|c|}{ DIPUTADOS ELECCIONES 2011} & \multicolumn{3}{|c|}{ DIPUTADOS ELECCIONES 2015} \\
\hline PP, 25 & PSOE, 23 & & PP, 16 & PSOE, 14 & PODEMOS, 3 \\
\hline \multicolumn{3}{|c|}{ Gobierno del PP con mayoría absoluta } & \multicolumn{3}{|c|}{ Gobierno del PSOE por pacto con PODEMOS } \\
\hline \multicolumn{6}{|c|}{ CORTES DE CASTILLA Y LEÓN } \\
\hline \multicolumn{3}{|c|}{ DIPUTADOS ELECCIONES 2011} & \multicolumn{3}{|c|}{ DIPUTADOS ELECCIONES 2015} \\
\hline PP, 53 & PSOE, 29 & $\mathrm{IU}, 1$ & PP, 42 & PSOE, 25 & PODEMOS, 10 \\
\hline UPL ${ }^{a}, 1$ & & & Cs, 5 & $\mathrm{IU}, 1$ & UPL, 1 \\
\hline \multicolumn{3}{|c|}{ Gobierno del PP con mayoría absoluta } & \multicolumn{3}{|c|}{ Gobierno del PP con mayoría simple } \\
\hline
\end{tabular}

\begin{tabular}{|c|c|c|c|c|c|c|c|c|c|c|c|}
\hline \multicolumn{12}{|c|}{ PARLAMENTO DE CATALUÑA } \\
\hline \multicolumn{3}{|c|}{$\begin{array}{c}\text { DIPUTADOS } \\
\text { ELECCIONES } 2010\end{array}$} & \multicolumn{3}{|c|}{$\begin{array}{l}\text { DIPUTADOS } \\
\text { ELECCIONES } 2012^{\mathrm{g}}\end{array}$} & \multicolumn{3}{|c|}{$\begin{array}{l}\text { DIPUTADOS } \\
\text { ELECCIONES } 2015^{j}\end{array}$} & \multicolumn{3}{|c|}{$\begin{array}{c}\text { DIPUTADOS } \\
\text { ELECCIONES } 2017\end{array}$} \\
\hline $\begin{array}{l}\mathrm{CIU}^{\mathrm{a}} \\
62\end{array}$ & $\begin{array}{l}\mathrm{PSC}^{\mathrm{b}} \\
28\end{array}$ & $\begin{array}{l}\text { PP, } \\
18\end{array}$ & $\begin{array}{c}\text { CIU, } \\
50\end{array}$ & $\begin{array}{c}\text { ERC, } \\
21\end{array}$ & $\begin{array}{c}\text { PSC, } \\
20\end{array}$ & JxSín, 62 & $\begin{array}{l}\text { Cs, } \\
25\end{array}$ & $\begin{array}{c}\text { PSC } \\
16\end{array}$ & Cs, 36 & $\begin{array}{l}\text { JUNTS } x \text { CATk } \\
34\end{array}$ & $\begin{array}{c}\text { ERC, } \\
\text { CATSI, } \\
32\end{array}$ \\
\hline $\begin{array}{c}\text { ICV- } \\
\text { EUiA }^{c} \text {, } \\
10\end{array}$ & $\begin{array}{l}\mathrm{ERC}^{\mathrm{d}} \\
\quad 10\end{array}$ & $\mathrm{Si}^{\mathrm{e}}, 4$ & $\begin{array}{l}\text { PP, } \\
19\end{array}$ & $\begin{array}{c}\text { ICV- } \\
\text { EUiA, } \\
13\end{array}$ & $\begin{array}{c}\text { Cs } \\
9\end{array}$ & $\begin{array}{c}\text { CATSÍQUEESPOT } \\
11\end{array}$ & $\begin{array}{c}\mathrm{PP} \\
11\end{array}$ & $\begin{array}{l}\text { CUP, } \\
10\end{array}$ & $\begin{array}{c}\text { PSC, } \\
17\end{array}$ & $\begin{array}{l}\text { CATCOMÚ- } \\
\text { PODEM, } 8\end{array}$ & CUP, 4 \\
\hline Cs, 3 & & & CUP $^{f}, 3$ & & & & & & PP, 4 & & \\
\hline \multicolumn{3}{|c|}{$\begin{array}{l}\text { Gobierno de } \mathrm{CIU} \text { con } \\
\text { mayoría simple }\end{array}$} & \multicolumn{3}{|c|}{$\begin{array}{l}\text { Gobierno de CIU con } \\
\text { pacto con ERC }\end{array}$} & \multicolumn{3}{|c|}{$\begin{array}{c}\text { Gobierno de Jx Sí por pacto } \\
\text { con la CUP }\end{array}$} & \multicolumn{3}{|c|}{$\begin{array}{l}\text { Gobierno de JUNTSxCAT en } \\
\text { minoría a partir del 17-05-2018 }\end{array}$} \\
\hline $\begin{array}{l}a \\
b \\
c \\
d \\
\text { e } \\
f \\
g \\
h \\
i \\
j \\
k\end{array}$ & $\begin{array}{l}\text { CONVERG } \\
\text { PARTIDO } \\
\text { INICIATIVA } \\
\text { ESQUERR } \\
\text { SOLIDARI } \\
\text { CANDIDAT } \\
\text { Se adelant } \\
\text { JUNTS PEI } \\
\text { CATALUNIA } \\
\text { Se adelanta } \\
\text { JUNTS PE }\end{array}$ & $\begin{array}{l}\text { SENCIA } \\
\text { SOCIAL } \\
\text { APER C } \\
\text { RA REP } \\
\text { TAT CA } \\
\text {-URA D } \\
\text { tan las } \\
\text { R SÍ = } \\
\text { A SII QU } \\
\text { an las e }\end{array}$ & $\begin{array}{l}\text { Y UNIÓN. } \\
\text { ISTA DE CA } \\
\text { ATALUNIA } \\
\text { JBLICANA } \\
\text { TALANA PE } \\
\text { UNITAT PO } \\
\text { lecciones } 2 \\
\text { onvergenci } \\
\text { E ES POT } \\
\text { ecciones } 14\end{array}$ & $\begin{array}{l}\text { TALUÑA } \\
\text { /EDS-ES } \\
\text { OE CATA } \\
\text { R LA IND } \\
\text { PULAR-A } \\
\text { años. } \\
\text { Democr } \\
\text { EQUO } \\
\text { meses. }\end{array}$ & $\begin{array}{l}\text { A. } \\
\text { SQUER } \\
\text { ALUÑA. } \\
\text { SEPEND } \\
\text { ALTERN } \\
\text { rática de } \\
\text { +EUiA + }\end{array}$ & $\begin{array}{l}\text { RA UNIDA I ALTERNATI } \\
\text { ENCIA. } \\
\text { JATIVA D'ESQUERRES } \\
\text { Cataluña (CDC) + ERC } \\
\text { ICV+ PODEM. } \\
\text { Europeo Catalán + CD }\end{array}$ & $\begin{array}{l}\text { IVA. } \\
\text { + Dem }\end{array}$ & ócratas & de Catal & $\tilde{n} a+$ Moviment $d^{\prime}$ & querres. \\
\hline
\end{tabular}


REALA. Nueva Época - N. ${ }^{\circ}$ 13, abril-septiembre 2020 - ISSN: 1989-8975 - DOI: 10.24965/reala.i13.10746 - [Págs. 131-151]

El impuesto sobre transmisiones patrimoniales y actos jurídicos documentados y el ciclo político: incidencia económica desde ... M. Ángeles Ortega Almón / Teresa Jesús Vílchez Ortiz / Araceli Rojo Gallego-Burin

\begin{tabular}{|c|c|c|c|c|c|}
\hline \multicolumn{6}{|c|}{ ASAMBLEA DE EXTREMADURA } \\
\hline \multicolumn{3}{|c|}{ DIPUTADOS ELECCIONES 2011} & \multicolumn{3}{|c|}{ DIPUTADOS ELECCIONES 2015} \\
\hline \multirow[t]{2}{*}{ PP, 32} & PSOE, 30 & IU, 3 & PSOE, 30 & PP, 28 & PODEMOS, 6 \\
\hline & & & Cs, 1 & & \\
\hline \multicolumn{3}{|c|}{ Gobierno del PP con mayoría simple } & \multicolumn{3}{|c|}{ Gobierno del PSOE por pacto con PODEMOS } \\
\hline \multicolumn{6}{|c|}{ PARLAMENTO DE LA XUNTA DE GALICIA } \\
\hline \multicolumn{3}{|c|}{ DIPUTADOS ELECCIONES 2012 ${ }^{a}$} & \multicolumn{3}{|c|}{ DIPUTADOS ELECCIONES 2016} \\
\hline PP, 41 & PSdeG $^{\mathrm{b}}, 18$ & $\mathrm{AGE}^{\mathrm{c}}, 9$ & PP, 41 & EN MAREA, 14 & PSdeG, 14 \\
\hline $\mathrm{BNG}^{\mathrm{d}}, 7$ & & & BNG-NÓS, 6 & & \\
\hline \multicolumn{3}{|c|}{ Gobierno del PP con mayoría absoluta } & \multicolumn{3}{|c|}{ Gobierno del PP con mayoría absoluta } \\
\hline \multicolumn{6}{|c|}{$\begin{array}{ll}\text { a } & \text { Se adelantan las elecciones } 6 \text { meses. } \\
\text { b } & \text { PARTIDO SOCIALISTA DE GALICIA. } \\
\text { c } & \text { ALTERNATIVA GALEGA DE ESQUERDA. } \\
\text { d } & \text { BLOQUE NACIONALISTA GALEGO. }\end{array}$} \\
\hline
\end{tabular}

\begin{tabular}{|c|c|c|c|c|c|}
\hline \multicolumn{6}{|c|}{ ASAMBLEA DE MADRID } \\
\hline \multicolumn{3}{|c|}{ DIPUTADOS ELECCIONES 2011} & \multicolumn{3}{|c|}{ DIPUTADOS ELECCIONES 2015} \\
\hline PP, 72 & PSOE, 36 & IU, 13 & PP, 48 & PSOE, 37 & PODEMOS, 27 \\
\hline UPyD, 8 & & & Cs, 17 & & \\
\hline \multicolumn{3}{|c|}{ Gobierno del PP con mayoría absoluta } & \multicolumn{3}{|c|}{ Gobierno del PP por pacto con CIUDADANOS } \\
\hline \multicolumn{6}{|c|}{ ASAMBLEA DE MURCIA } \\
\hline \multicolumn{3}{|c|}{ DIPUTADOS ELECCIONES 2011} & \multicolumn{3}{|c|}{ DIPUTADOS ELECCIONES 2015} \\
\hline \multirow[t]{2}{*}{ PP, 33} & PSOE, 11 & $\mathrm{IU}, 1$ & PP, 22 & PSOE, 13 & PODEMOS, 6 \\
\hline & & & Cs, 4 & & \\
\hline \multicolumn{3}{|c|}{ Gobierno del PP con mayoría absoluta } & \multicolumn{3}{|c|}{ Gobierno del PP por pacto con CIUDADANOS } \\
\hline \multicolumn{6}{|c|}{ PARLAMENTO DE LA RIOJA } \\
\hline \multicolumn{3}{|c|}{ DIPUTADOS ELECCIONES 2011} & \multicolumn{3}{|c|}{ DIPUTADOS ELECCIONES 2015} \\
\hline \multirow[t]{2}{*}{ PP, 17} & PSOE, 14 & $\mathrm{PR}^{\mathrm{a}}, 2$ & PP, 15 & PSOE, 14 & PODEMOS, 4 \\
\hline & & & Cs, 4 & & \\
\hline \multicolumn{3}{|c|}{ Gobierna el PP con mayoría absoluta } & \multicolumn{3}{|c|}{ Gobierna el PP con mayoría simple } \\
\hline
\end{tabular}

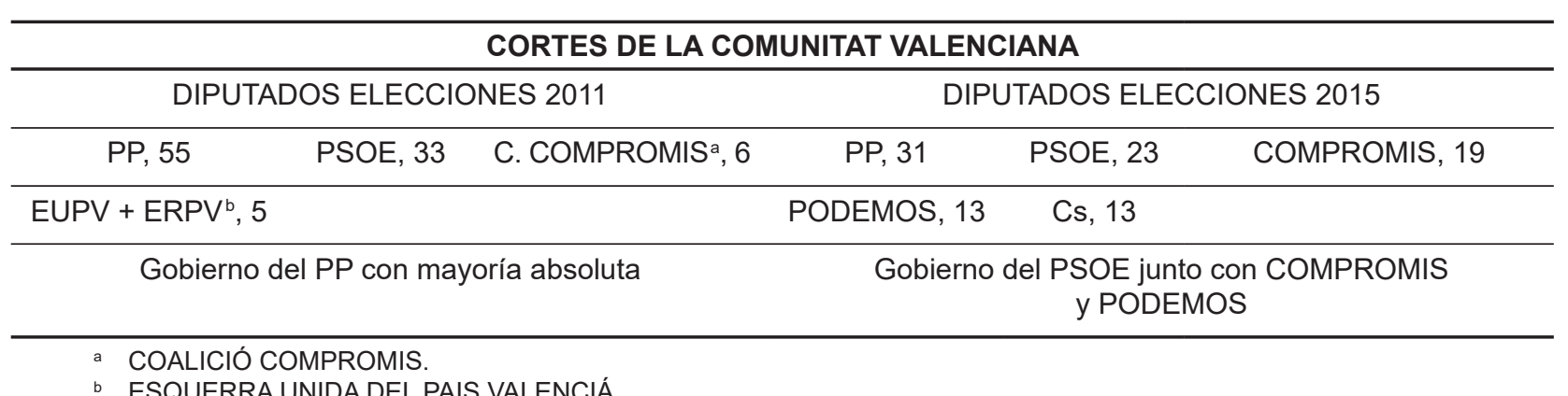

b ESQUERRA UNIDA DEL PAIS VALENCIÁ.

Fuente: Junta Electoral Central y elaboración propia. 
REALA. Nueva Época - N. 13, abril-septiembre 2020 - ISSN: 1989-8975 - DOI: 10.24965/reala.i13.10746 - [Págs. 131-151]

El impuesto sobre transmisiones patrimoniales y actos jurídicos documentados y el ciclo político: incidencia económica desde .. M. Ángeles Ortega Almón / Teresa Jesús Vílchez Ortiz / Araceli Rojo Gallego-Burin

\section{REFERENCIAS BIBLIOGRÁFICAS}

AGENCIA ESTATAL DE LA ADMINISTRACIÓN TRIBUTARIA (2019): Tipos Impositivos en el IVA. Fecha de consulta: 15-05-2019. URL: https://www.agenciatributaria.es/static_files/AEAT/Contenidos_Comunes/La_Agencia_ Tributaria/Segmentos_Usuarios/Empresas y_profesionales/Novedades_IVA_2014/Nuevos_tipos_IVA.pdf.

AGENCIA TRIBUTARIA DE CATALUÑA (2019): Guía Práctica Impuesto sobre Transmisiones Patrimoniales y Actos Jurídicos Documentados (ITPAJD). Fecha de consulta: 05-03-2019. URL: https://atc.gencat.cat/web/.content/ documents/02_doc_tributs/01_itpajd/guia_itp_es.pdf.

ALVAREDO, F.; SÁEZ, E. (2009): "Income and wealth concentration in Spain from a historical and fiscal perspective", en Journal of the European Economic Association, vol. 7, núm. 5, págs. 1.140-1.167. URL: https://onlinelibrary. wiley.com/doi/abs/10.1162/JEEA.2009.7.5.1140. DOI: $h$ ttps://doi.org/10.1162/JEEA.2009.7.5.1140.

CENTRO DE ESTUDIOS FINANCIEROS (CEF) (2019): "El Impuesto sobre Transmisiones Patrimoniales y Actos Jurídicos Documentados. Cuestiones Generales", en CENTRO DE ESTUDIOS FINANCIEROS (CEF): Guía Fiscal 2019, cap. 6. Madrid: Centro de Estudios Financieros (CEF). Fecha de consulta: 04-03-2019. URL: https://www.fiscal-impuestos.com/guia-fiscal-capitulo-6-impuesto-transmisiones-patrimoniales-actos-juridicosdocumentados-cuestiones-generales.

CONSEJO DE POLÍTICA FISCAL Y FINANCIERA (2009): Acuerdo 6/2009 de 15 de julio para la Reforma del Sistema de Financiación de las Comunidades Autónomas de Régimen Común y Ciudades con Estatuto de Autonomía. Fecha de consulta: 23-05-2019. URL: https://www.hacienda.gob.es/Documentacion/Publico/PortalVarios/ FinanciacionTerritorial/Autonomica/AcuerdosConsejo/Acuerdo\%206_2009\%20Reforma\%20Sistema\%20 Financiaci\%C3\%B3n.pdf.

JUNTA ELECTORAL CENTRAL (2019): Elecciones Autonómicas (Todas las Comunidades Autónomas de Régimen Común). Fecha de consulta: 08-04-2019. URL: http://www.juntaelectoralcentral.es/cs/jec/elecciones/autonomicas.

MARTÍNEZ SÁNCHEZ, C. (2018): “¿Tiene sentido que exista un impuesto sobre la riqueza?”, en Razón y Fe, vol. 277, núm. 1.432, págs. 159-171. Fecha de consulta: 22-04-2019. URL: https://revistas.comillas.edu/index.php/ razonyfe/article/view/9115.

MINISTERIO DE HACIENDA (2017): Reforma del Sistema de Financiación Autonómica. Informe de la Comisión de Expertos para la Revisión del Modelo de Financiación Autonómica. Fecha de consulta: 03-04-2018. URL: https:// www.hacienda.gob.es/es-ES/CDI/Paginas/SistemasFinanciacionDeuda/InformacionCCAAs/Reforma_SFA.aspx. URL: https://www.hacienda.gob.es/CDI/sist\%20financiacion\%20y\%20deuda/informaci\%C3\%B3nccaa/informe_ final_comisi\%C3\%B3n_reforma_sfa.pdf.

MINISTERIO DE HACIENDA (2016): Haciendas Autonómicas en cifras. Las Haciendas Autonómicas en cifras 2016. Fecha de consulta: 16-04-2019. URL: https://www.hacienda.gob.es/CDI/Sist\%20Financiacion\%20y\%20Deuda/ Informaci\%C3\%B3nCCAA/Las_Haciendas_Auton\%C3\%B3micas_en_cifras_2016.pdf.

MINISTERIO DE HACIENDA (2015): Haciendas Autonómicas en cifras. Las Haciendas Autonómicas en cifras 2015. Fecha de consulta: 16-04-2019. URL: https://www. hacienda.gob.es/Documentacion/Publico/CDI/ Sist\%20Financiacion\%20y\%20Deuda/Informaci\%C3\%B3nCCAA/Las_Haciendas_Auton\%C3\%B3micas_en_ cifras_2015.pdf.

MINISTERIO DE HACIENDA (2014): Haciendas Autonómicas en cifras. Las Haciendas Autonómicas en cifras 2014. Fecha de consulta: 16-04-2019. URL: https://www.hacienda.gob.es/Documentacion/Publico/CDI/ Sist\%20Financiacion\%20y\%20Deuda/Informaci\%C3\%B3nCCAA/Las_Haciendas_Auton\%C3\%B3micas_en_ cifras_2014.pdf.

MINISTERIO DE HACIENDA (2013): Haciendas Autonómicas en cifras. Las Haciendas Autonómicas en cifras 2013. Fecha de consulta: 16-04-2019. URL: https://www.hacienda.gob.es/Documentacion/Publico/PortalVarios/ FinanciacionTerritorial/Autonomica/LasHaciendasAuton\%C3\%B3micasEnCifras2013.pdf.

MINISTERIO DE HACIENDA (2012): Haciendas Autonómicas en cifras. Las Haciendas Autonómicas en cifras 2012. Fecha de consulta: 16-04-2019. URL: https://www.hacienda.gob.es/Documentacion/Publico/PortalVarios/ FinanciacionTerritorial/Autonomica/LasHaciendasAutonómicasEnCifras2012.pdf.

MINISTERIO DE HACIENDA (2011): Haciendas Autonómicas en cifras. Las Haciendas Autonómicas en cifras 2011. Fecha de consulta: 16-04-2019. URL: https://www.hacienda.gob.es/Documentacion/Publico/PortalVarios/ FinanciacionTerritorial/Autonomica/SECCIONES\%201\%20II\%20Y\%20II\%20HH\%20AA\%202011\%20definitivo.pdf.

MINISTERIO DE HACIENDA (2010): Haciendas Autonómicas en cifras. Las Haciendas Autonómicas en cifras 2010. Fecha de consulta: 16-04-2019. URL: https://www.hacienda.gob.es/Documentacion/Publico/PortalVarios/ FinanciacionTerritorial/Autonomica/SECCIONES\%20\%201\%20\%20II\%20\%20Y\%20III\%20\%20HH\%20AA\%20 2010.pdf.

MINISTERIO DE HACIENDA (2009): Haciendas Autonómicas en cifras. Las Haciendas Autonómicas en cifras 2009. Fecha de consulta: 16-04-2019. URL: https://www.hacienda.gob.es/Documentacion/Publico/PortalVarios/ FinanciacionTerritorial/Autonomica/SECCIONES\%201\%2011\%20II\%20HH\%20AA\%202009.pdf.

MINISTERIO DE HACIENDA (2008): Haciendas Autonómicas en cifras. Las Haciendas Autonómicas en cifras 2008. Fecha de consulta: 16-04-2019. URL: https://www.hacienda.gob.es/Documentacion/Publico/PortalVarios/ FinanciacionTerritorial/Autonomica/LAS\%20HACIENDAS\%20AUTONOMICAS\%20EN\%20CIFRAS\%202008.pdf. 
REALA. Nueva Época - N. ${ }^{13}$, abril-septiembre 2020 - ISSN: 1989-8975 - DOI: 10.24965/reala.i13.10746 - [Págs. 131-151]

El impuesto sobre transmisiones patrimoniales y actos jurídicos documentados y el ciclo político: incidencia económica desde ... M. ${ }^{a}$ Ángeles Ortega Almón / Teresa Jesús Vílchez Ortiz / Araceli Rojo Gallego-Burin

MINISTERIO DE HACIENDA (2007): Haciendas Autonómicas en cifras. Las Haciendas Autonómicas en cifras 2007. Fecha de consulta: 16-04-2019. URL: https://www.hacienda.gob.es/Documentacion/Publico/Porta/Varios/ FinanciacionTerritorial/Autonomica/LAS\%20HACIENDAS\%20AUTONOMICAS\%20EN\%20CIFRAS\%202007.pdf.

MINISTERIO DE HACIENDA (2006): Haciendas Autonómicas en cifras. Las Haciendas Autonómicas en cifras 2006. Fecha de consulta: 16-04-2019. URL: https://www.hacienda.gob.es/Documentacion/Publico/PortalVarios/ FinanciacionTerritorial/Autonomica/LAS\%20HACIENDAS\%20AUTONOMICAS\%20EN\%20CIFRAS\%202006.pdf.

MINISTERIO DE HACIENDA (2019): Recaudación definitiva por tributos cedidos. Recaudación definitiva por tributos cedidos 2017. Fecha de consulta: 20-04-2019. URL: https://www.hacienda.gob.es/CDI/impuestos/ recaudaciondefinitiva2017.pdf.

REAF-REGAF ASESORES FISCALES DEL CONSEJO GENERAL DE ECONOMISTAS DE ESPAÑA (2009): Panorama de la Fiscalidad Autonómica 2009. URL: https://baobab.uc3m.es/monet/monnet/IMG/pdf/Panorama_Fiscalidad_ CCAA_2009.pdf.

REAF-REGAF ASESORES FISCALES DEL CONSEJO GENERAL DE ECONOMISTAS DE ESPAÑA (2010): Panorama de la Fiscalidad Autonómica 2010. URL: http://estaticos.expansion.com/estaticas/documentos/2010/03/ documento12032010.pdf.

REAF-REGAF ASESORES FISCALES DEL CONSEJO GENERAL DE ECONOMISTAS DE ESPAÑA (2011): Panorama de la Fiscalidad Autonómica 2011. URL: https://www.diariojuridico.com/wp-content/uploads/kalins-pdf/singles/elreaf-presenta-el-panorama-de-la-fiscalidad-autonomica-2011.pdf.

REAF-REGAF ASESORES FISCALES DEL CONSEJO GENERAL DE ECONOMISTAS DE ESPAÑA (2012): Panorama de la Fiscalidad Autonómica 2012. URL: https://economistas.es/Contenido/REAF/Bofiper/PANORAMA\%20 FISCALIDAD\%20AUTONOMICA\%20Y\%20FORAL\%202012.pdf.

REAF-REGAF ASESORES FISCALES DEL CONSEJO GENERAL DE ECONOMISTAS DE ESPAÑA (2013): Panorama de la Fiscalidad Autonómica y Foral 2013. URL: http://gestrisam.malaga.eu/portal/menu/seccion_0010/ documentos/Panorama_20CCAA_202013.pdf.

REAF-REGAF ASESORES FISCALEES DEL CONSEJO GENERAL DE ECONOMISTAS DE ESPAÑA (2014): Panorama de la Fiscalidad Autonómica y Foral 2014. URL: http://s01.s3c.es/imag/doc/2014-02-27/16.Fiscal.pdf.

REAF-REGAF ASESORES FISCALES DEL CONSEJO GENERAL DE ECONOMISTAS DE ESPAÑA (2015): Panorama de la Fiscalidad Autonómica y Foral 2015. URL: http://www.lorenteylorente.es/2015/03/panorama-de-la-fiscalidadautonomica-en-2015/.

REAF-REGAF ASESORES FISCALES DEL CONSEJO GENERAL DE ECONOMISTAS DE ESPAÑA (2016): Panorama de la Fiscalidad Autonómica y Foral 2016. URL: https://www.economistas.es/Contenido/REAF/Informes/REAFPanorama2016WEB.pdf.

REAF-REGAF ASESORES FISCALES DEL CONSEJO GENERAL DE ECONOMISTAS DE ESPAÑA (2017): Panorama de la Fiscalidad Autonómica y Foral 2017. URL: https://www.economistas.es/Contenido/REAF/ NOTAS\%20PRENSA/PANORAMA\%20FISCALIDAD\%20CCAA\%202017.pdf.

REAF-REGAF ASESORES FISCALES DEL CONSEJO GENERAL DE ECONOMISTAS DE ESPAÑA (2018): Panorama de la Fiscalidad Autonómica y Foral 2018. URL: https://www.economistas.es/Contenido/Consejo/Estudios\%20 y\%20trabajos/REAF-\%20Panorama\%20de\%20la\%20Fiscalidad\%20CCAA-2018.pdf.

REAF-REGAF ASESORES FISCALES DEL CONSEJO GENERAL DE ECONOMISTAS DE ESPAÑA (2019): Panorama de la Fiscalidad Autonómica y Foral 2019. URL: https://www.economistas.es/Contenido/Consejo/Estudios\%20 y\%20trabajos/Panorama\%20Fiscalidad\%20CCAA\%202019.pdf. 